\title{
PENTA-GRAPHENE: A NEW CARBON ALLOTROPE
}

\author{
1,2,3Shunhong Zhang, ${ }^{3}$ Jian Zhou, ${ }^{1,2,3}$ Qian Wang, ${ }^{4,5}$ Xiaoshuang Chen, ${ }^{6}$ Yoshiyuki \\ Kawazoe, ${ }^{3}$ Puru Jena \\ ${ }^{1}$ Center for Applied Physics and Technology, College of Engineering, Peking University, Beijing 100871, China; \\ ${ }^{2}$ Collaborative Innovation Center of Inertial Fusion Sciences and Applications, Ministry of Education, Beijing \\ 100871, China; \\ ${ }^{3}$ Department of Physics, Virginia Commonwealth University, Richmond, VA 23284; \\ ${ }^{4}$ National Laboratory for Infrared Physics, Shanghai Institute of Technical Physics, Chinese Academy of \\ Sciences, Shanghai 200083, China; \\ ${ }^{5}$ Synergetic Innovation Center of Quantum Information \& Quantum Physics, University of Science and \\ Technology of China, Hefei, Anhui 230026, China; \\ ${ }^{6}$ Institute for Materials Research, Tohoku University, Sendai, 980-8577, Japan \\ qianwang2@pku.edu.cn, kawazoe@imr.edu
}

A 2D metastable carbon allotrope, penta-graphene, composed entirely of carbon pentagons and resembling the Cairo pentagonal tiling, is proposed. State-of-the-art theoretical calculations confirm that the new carbon polymorph is not only dynamically and mechanically stable, but also can withstand temperatures as high as $1000 \mathrm{~K}$. Due to its unique atomic configuration, penta-graphene has an unusual negative Poisson's ratio and ultrahigh ideal strength that can even outperform graphene. Furthermore, unlike graphene that needs to be functionalized for opening a band gap, penta-graphene possesses an intrinsic quasi-direct band gap as large as $3.25 \mathrm{eV}$, close to that of $\mathrm{ZnO}$ and GaN. Equally important, penta-graphene can be exfoliated from T12-carbon. When rolled up, it can form pentagon-based nanotubes which are semiconducting, regardless of their chirality. When stacked in different patterns, stable 3D twin structures of T12-carbon are generated with band gaps even larger than that of T12-carbon. The versatility of penta-graphene and its derivatives are expected to have broad applications in nanoelectronics and nanomechanics.

Key-words: carbon allotrope, carbon pentagon, stability, negative Poisson's ratio, electronic structure

PACS: 51.05.ue

Bibliography - 54 references

Received 15.11.2015

RENSIT, 2015, 7(2)191-207

CONTENTS

1. INTRODUCTION (191)

2. Results (192)

2.1. Penta-Graphene Exfoliated from T12-Carbon (192)

2.2. Energetic Stability (193)

2.3. Dynamic Stability (193)

2.4. Thermal Stability (194)

2.5. Mechanical Stability (194)

2.6. Mechanical Properties (195)

2.7. Electronic Properties (96)

2.8. Penta-Tubes: Rolled-Up Penta-Graphene (197)

2.9. 3D Carbon Structures: Stacked PentaGraphene Layers (197)

3. Discussion (198)

4. Methods (199)

5. SI APpendix (200)

ACKNOWLEDgMents (206)

References (206)

\section{INTRODUCTION ${ }^{1}$}

DOI: $10.17725 /$ rensit.2015.07.191

Carbon is one of the most versatile elements in the periodic table and forms a large number of allotropes ranging from the well-known graphite, diamond, $C_{60}$ fullerene [1], nanotube [2], and graphene [3] to the newly discovered carbon nanocone [4], nanochain [5], graphdiyne [6], as well as $3 D$ metallic structures [7, 8]. The successful synthesis of graphene [3] has triggered considerable interest in exploring novel carbon-based nanomaterials. A wealth of $2 D$ carbon allotropes beyond graphene has since been studied (see SI Appendix, Table S1 for details). Although some of these polymorphs such as graphdiyne [6] are metastable compared with graphene, they have

\footnotetext{
${ }^{1}$ Author contributions: Q.W. designed research; S.Z. and J.Z. performed research; S.Z., J.Z., Q.W., X.C., Y.K., and P.J. analyzed data; and S.Z., J.Z., Q.W., X.C., Y.K., and P.J. wrote the paper.
} 
been successfully synthesized. Moreover, some 2D carbon allotropes are predicted to exhibit remarkable properties that even outperform graphene, such as anisotropic Dirac cones [9], inherent ferromagnetism [10], high catalytic activity [6], and potential superconductivity related to the high density of states at the Fermi level [11]. These results demonstrate that many of the novel properties of carbon allotropes are intimately related to the topological arrangement of carbon atoms and highlight the importance of structure-property relationships [12].

Pentagons and hexagons are two basic building blocks of carbon nanostructures. From zerodimensional nanoflakes or nanorings [13] to $1 D$ nanotube, $2 D$ graphene, and $3 D$ graphite and metallic carbon phases [7, 8], hexagon is the only building block. Extended carbon networks composed of only pentagons are rarely seen. Carbon pentagons are usually considered as topological defects or geometrical frustrations [14] as stated in the well-known "isolated pentagon rule" (IPR) [15] for fullerenes, where pentagons must be separated from each other by surrounding hexagons to reduce the steric stress. For instance, $C_{60}$ consists of 12 pentagons separated by 20 hexagons forming the shape of a soccer ball, which is a perfect footnote to IPR. The emergence of carbon pentagons is also found to be accompanied by carbon heptagons in some cases [14], but are separated from each other. Inspired by the synthesis of pure pentagon-based $C_{20}$ cage (16), considerable effort has been made to stabilize fusedpentagon-based and non-IPR carbon materials in various dimensionalities $[10,15]$. Some non-IPR fullerenes have been experimentally realized [15]. A "pentagon-first" mechanism was postulated in the transformation from $s p$ carbon chains to $s p^{2}$ carbon rings during surface growth of $2 D$ carbon sheets [17]. Thus, we conceived the idea of building $2 D$ carbon sheets using fused pentagons as a structural motif. In this work, we show that a $2 D$ carbon allotrope, penta-graphene, consisting entirely of pentagons, can indeed exist. The dynamical, thermal, and mechanical stability of this unique structure is confirmed by a series of state-of-theart theoretical calculations. In addition, we show that pentagon-based carbon nanotubes, penta-tubes, formed by rolling up the penta-graphene sheet, and $3 D$ twin structures of the recently reported T12-carbon [18] formed by stacking these sheets in different patterns, are both dynamically and thermally stable. We demonstrate that these exotic pentagon-based carbon materials exhibit interesting mechanical and electronic properties ${ }^{2}$.

\section{RESULTS}

2.1. Penta-Graphene Exfoliated from T12Carbon. Our search for an allpentagon-based $2 D$ carbon sheet began by examining the recently proposed T12-carbon phase [18] that can be acquired by heating an interlocking-hexagon-based metastable carbon phase at high temperature [8]. We note that there are two kinds of $C-C$ bond lengths, namely, the slightly shorter intralayer bond $\left(d_{1}\right)$ and the slightly longer interlayer bond $\left(d_{2}\right)$, as shown in Fig. 1A. Here the atoms displayed in ball-stick model and highlighted in yellow form a layered structure which can be chemically exfoliated from the T12-carbon phase. In fact, a monolayer can be fabricated from either a layered structure or a nonlayered structure [19]. For van der Waals coupled layered structures like graphite, mechanical exfoliation [3] is sufficient to obtain the monolayer sheet (graphene), whereas for chemically bonded bulk phases like MAX (a family of transition metal carbides or nitrides), chemical exfoliation technique has been developed to extract a single layer (MXene) [20].

The optimized crystal structure of the $2 D$ pentagon-based phase generated by exfoliating a single layer from T12-carbon is shown in Fig. 1B. The structure possesses $\mathrm{P}-42, \mathrm{~m}$ symmetry (space group no. 113) with a tetragonal lattice. The optimized lattice constants are $a=b=3.64 \AA$. The top view shows that the new phase is composed entirely of carbon pentagons, forming a beautiful pattern well known as Cairo pentagonal tiling [21]. From the side view a buckling $(0.6 \AA)$ is observed, leading to a $2 D$ sheet with_ a total thickness of $1.2 \AA$. This structure 'Significance

Carbon has many faces - from diamond and graphite to graphene, nanotube, and fullerenes. Whereas hexagons are the primary building blocks of many of these materials, except for $C_{20}$ fullerene, carbon structures made exclusively of pentagons are not known. Because many of the exotic properties of carbon are associated with their unique structures, some fundamental questions arise: Is it possible to have materials made exclusively of carbon pentagons and if so will they be stable and have unusual properties? Based on extensive analyses and simulations we show that penta-graphene, composed of only carbon pentagons and resembling Cairo pentagonal tiling, is dynamically, thermally, and mechanically stable. It exhibits negative Poisson's ratio, a large band gap, and an ultrahigh mechanical strength. 

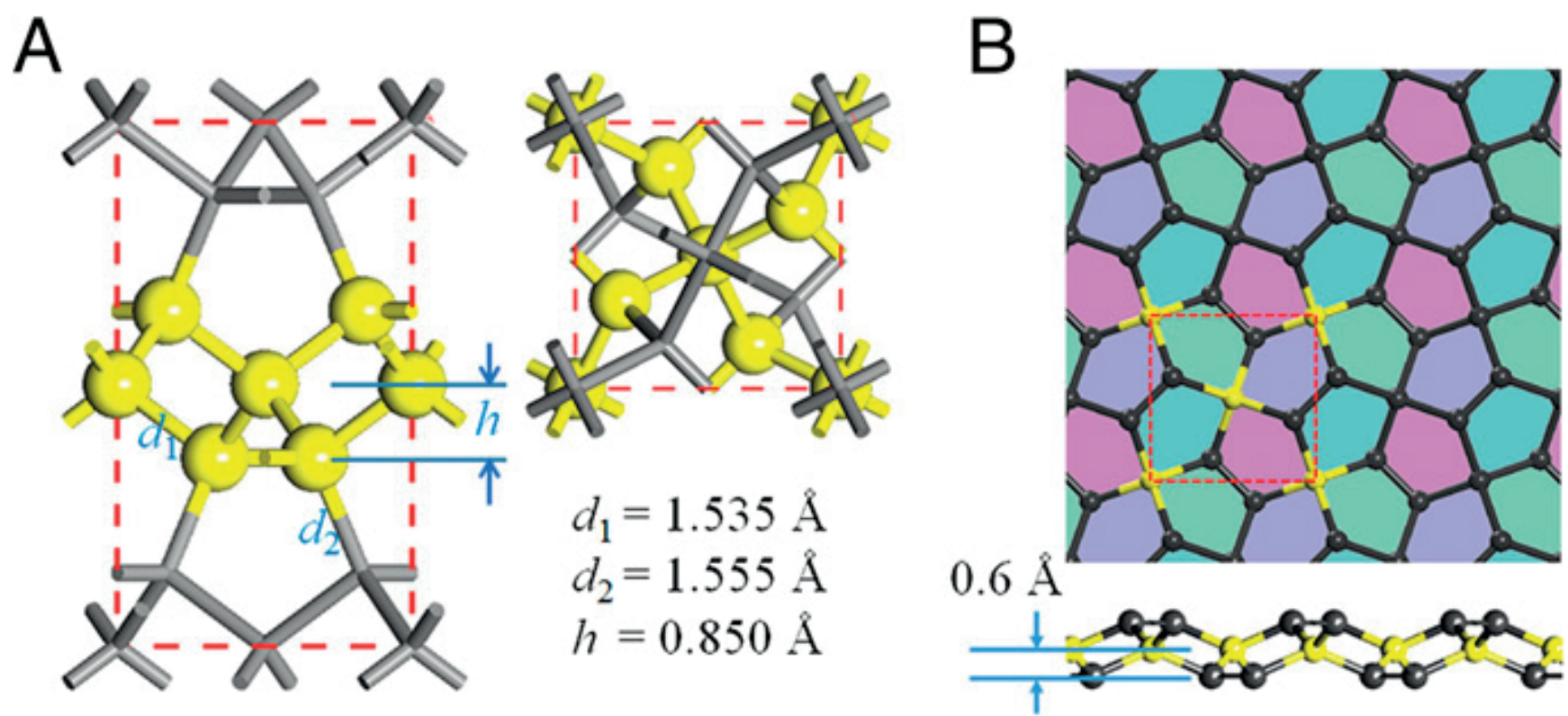

Fig. 1. (A) Crystal structure of T12-carbon viewed from the [100] and [001] directions, respectively. (B) Top and side views of the atomic configuration of penta-graphene. The square marked by red dashed lines denotes a unit cell, and the highlighted balls represent the sp ${ }^{3}$ bybridized $C$ atoms.

can be considered as a multidecker sandwich, with the 4-coordinated $C$ atoms highlighted in yellow in Fig. 1B sandwiched between the 3-coordinated atoms. For convenience of discussion, we hereafter group the $s p^{3}$ - and $s p^{2}$-hybridized $C$ atoms as $C 1$ and $C 2$, respectively, and call this new graphene allotrope penta-graphene. The unit cell of penta-graphene contains six atoms as denoted by red dashed lines in Fig. $1 \mathrm{~B}$ in which the $C 1$ to $C 2$ ratio is $1: 2$. The $C 1-$ $C 2(1.55 \AA)$ and $C 2-C 2(1.34 \AA)$ bond lengths show pronounced characters of single and double bonds, respectively, and the bond angle $\theta_{C 2-C 1-C 2}$ is $134.2^{\circ}$, indicating the distorted $s p^{3}$ character of $C 1$ atoms. Such bond multiplicity [22] of carbon, although absent in the well-known diamond, graphite and graphene, has been found in a number of carbon structures with different dimensionalities [6-8, 22, 23 and is of general chemical interest as it leads to intermediate valency [23]. Interestingly, we note that penta-graphene resembles the structure of experimentally identified layered silver azide $\left(A g N_{3}\right)$ [24]. By replacing the $N_{3}$ moieties and $A g$ atoms with the triconnected $C$ dimers and tetra-connected $C$ atoms, respectively, the geometry of pentagraphene can be realized.

2.2. Energetic Stability. Total energy calculations are performed to investigate the thermodynamic stability of penta-graphene. Although this phase is metastable compared with graphene and previously reported $2 D$ carbon allotropes $[6,11,14]$ due to its violation of the IPR, it is more stable than some nanoporous carbon phases such as $3 D T$-carbon [25], 2D $\alpha$-graphyne [6], and $(3,12)$-carbon sheet [26] (Fig. 2A). We also note that pentagraphene is energetically preferable over some experimentally identified carbon nanostructures such as the smallest fullerene, $C_{20}$ and the smallest carbon nanotube, implying that the $2 D$ penta-graphene sheet could be synthesized. Although $C_{20}$ cage and penta-graphene share the structural motif of fused pentagons, unlike the highly curved $C_{20}$ cage where all of the $C$ atoms exhibit distorted $s p^{2}$ hybridization leading to a large strain energy, in penta-graphene the onset of $s p^{3}$ hybridization lowers the curvature of fused carbon pentagons, thus partially releasing the strain.

2.3. Dynamic Stability. Next we study the lattice dynamics of penta-graphene by calculating its phonon dispersion. The results are presented in Fig. 2B. The absence of imaginary modes in theentire Brillouin zone confirms that penta-graphene is dynamically stable. Similar to the phonons of graphene [27, 28], there are three distinct acoustic modes in the phonon spectra of penta-graphene. The in-plane longitudinal and in-plane transverse modes have linear dispersion near the $\Gamma$ point, whereas the outof-plane (ZA) mode has quadratic dispersion when $\mathbf{q}$ approaches $\mathbf{0}$. The quadratic ZA mode in the long-wavelength region is closely associated with the bending rigidity and lattice heat capacity of the nanosheet, which is discussed in detail in SI Appendix, 
text S1. A remarkable phonon gap is observed in the phonon spectra. Detailed analysis of the atomresolved phonon density of states (PhDOS) reveals that the double bonds between the $s p^{2}$ hybridized $C 2$ atoms are predominant in the dispersionless highfrequency modes (Fig. 2B), which is quite similar to the phonon modes in earlier reported $s p^{2}-s p^{3}$ hybrid carbon structures $[7,8]$.

2.4. Thermal Stability. The thermal stability of penta-graphene is examined by performing ab initio molecular dynamics (AIMD) simulations using canonical ensemble. To reduce the constraint of periodic boundary condition and explore possible structure reconstruction, the $2 D$ sheet is simulated by $(4 \times 4),(6 \times 6)$, and $(4 \sqrt{ } 2 \times 4 \sqrt{ } 2) \mathrm{R} 45^{\circ}$ supercells, respectively. After heating at room temperature (300 K) for 6 ps with a time step of $1 \mathrm{fs}$, no structure reconstruction is found to occur in all of the cases. Furthermore, we find that the penta-graphene sheet can withstand temperatures as high as 1,000 $\mathrm{K}$, implying that this $2 \mathrm{D}$ carbon phase is separated by high-energy barriers from other local minima on the potential energy surface (PES) of elemental carbon. The snapshots of atomic configurations of penta-graphene at the end of AIMD simulations are shown in SI Appendix, Fig. S1. The effect of point defects or rim atoms on the stability of the penta-graphene sheet is also studied by introducing mono- and di-vacancies, Stone-Wales-like defect, adatoms, and edge atoms. The results are presented in SI Appendix, text S2, Figs. S2-S4, where one can

A

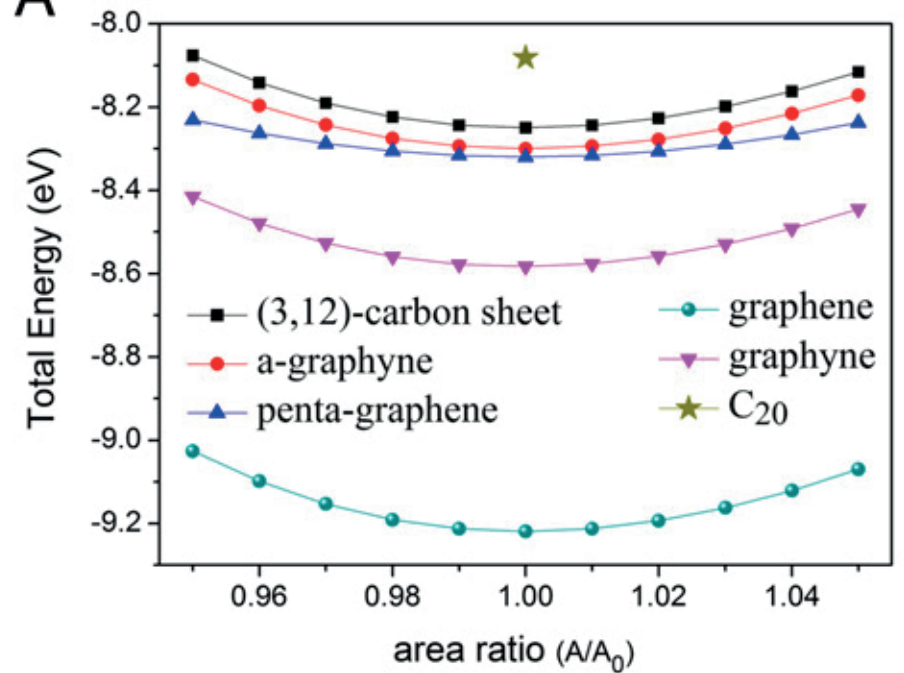

see that the stability and structure of penta-graphene is robust, despite the defects.

2.5. Mechanical Stability. As we fix the supercell during all of the MD simulations, it is necessary to assess the effect of lattice distortion on structural stability. To guarantee the positive-definiteness of strain energy following lattice distortion, the linear elastic constants of a stable crystal has to obey the Born-Huang criteria [29]. We calculate the change of energy due to the in-plane strain to determine the mechanical stability of penta-graphene. For a $2 D$ sheet, using the standard Voigt notation [26], i.e., $1-x x, 2-y y$, and $6-x y$, the elastic strain energy per unit area can be expressed as

$U(\varepsilon)=\frac{1}{2} C_{11} \varepsilon_{x x}^{2}+\frac{1}{2} C_{22} \varepsilon_{y y}^{2}+C_{12} \varepsilon_{x x} \varepsilon_{y y}+2 C_{66} \varepsilon_{x y}^{2}$,

where $C_{11}, C_{22}, C_{12}$, and $C_{66}$ are components of the elastic modulus tensor, corresponding to second partial derivative of strain energy with respect to strain. The elastic constants can be derived by fitting the energy curves associated with uniaxial and equibiaxial strains. The curves are plotted in SI Appendix, Fig. S5. For a mechanically stable $2 D$ sheet [29], the elastic constants need to satisfy $C_{11} C_{22}-$ $C_{12}{ }^{2}>0$ and $C_{66}>0$. Due to the tetragonal symmetry of penta-graphene, we have $C_{11}=C_{22}$. Thus, in this case we only need to satisfy $C_{11}>\left|C_{12}\right|$ and $C_{66}>$ 0 . Under uniaxial strain, $\varepsilon_{\mathrm{yy}}=0, \mathrm{U}(\varepsilon)=(1 / 2) C_{11} \varepsilon_{11}{ }^{2}$. Parabolic fitting of the uniaxial strain curve yields $C_{11}=265 \mathrm{GPa} \cdot \mathrm{nm}$. Under equi-biaxial strain, $\varepsilon_{\mathrm{xx}}=$ $\varepsilon_{\mathrm{vy}}$, we have $\mathrm{U}(\varepsilon)=\left(C_{11}+C_{12}\right) \varepsilon_{\mathrm{xx}}{ }^{2}$. Again, by fitting the equi-biaxial strain curve we obtain $C_{11}+C_{12}=$

B

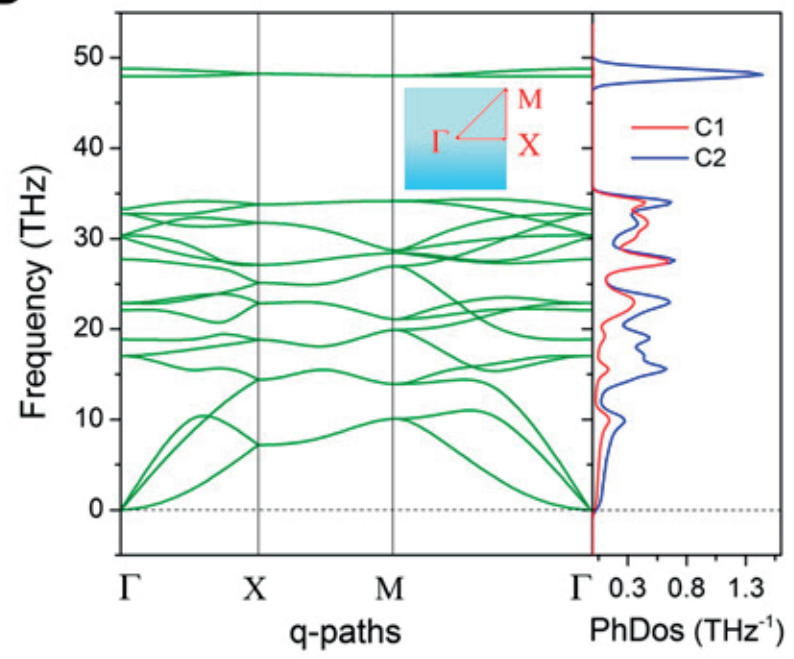

Fig. 2. (A) Area dependence of total energy per atom for some 2D carbon allotropes. The total energy of the experimentally identified dodecahedral $C_{20}$ cage is also calculated and plotted here for comparison. (B) Phonon band structures and PhDOS of penta-graphene. (Inset) High-symmetric q-point paths: $\Gamma(0,0) \rightarrow X(1 / 2,0) \rightarrow M(1 / 2,1 / 2) \rightarrow \Gamma(0,0)$. 
$247 \mathrm{GPa} \cdot \mathrm{nm}$, hence, $C_{12}=-18 \mathrm{GPa} \cdot \mathrm{nm}$. Thus, the calculated elastic constants satisfy $C_{11}>\left|C_{12}\right|$, and the calculated $C_{66}$ is positive, indicating that the $2 \mathrm{D}$ pentagraphene sheet is mechanically stable.

2.6. Mechanical Properties. Having confirmed the stability of pentag-raphene, we next systematically study its mechanical properties. The in-plane Young's modulus, which can be derived from the elastic constants by $\mathrm{E}=\left(C_{11}{ }^{2}-C_{12}{ }^{2}\right)=C_{11}$, is calculated to be 263.8 GPa.nm, which is more than two-thirds of that of graphene (345 GPanm) [30] and is comparable to that of $h-B N$ monolayer [26]. Interestingly, we note that $C_{12}$ is negative for this nanosheet, leading to a negative Poisson's ratio (NPR), viz., $\nu_{12}=v_{21}=$ $C_{12} / C_{11}=-0.068$. To confirm this unusual result, we calculated the lateral response in the $y$ direction when the lattice endures a tensile strain in the $x$

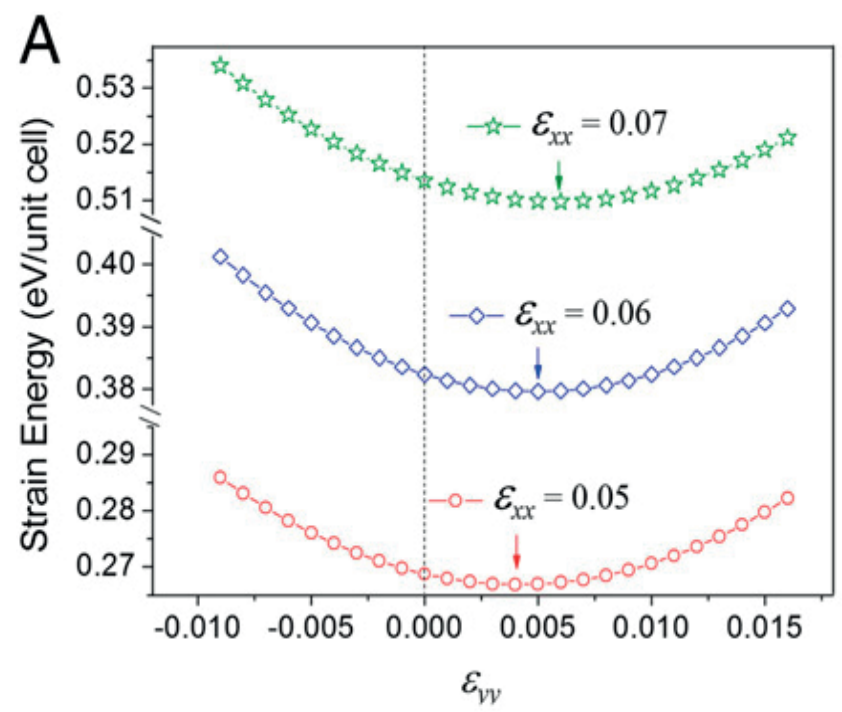

C

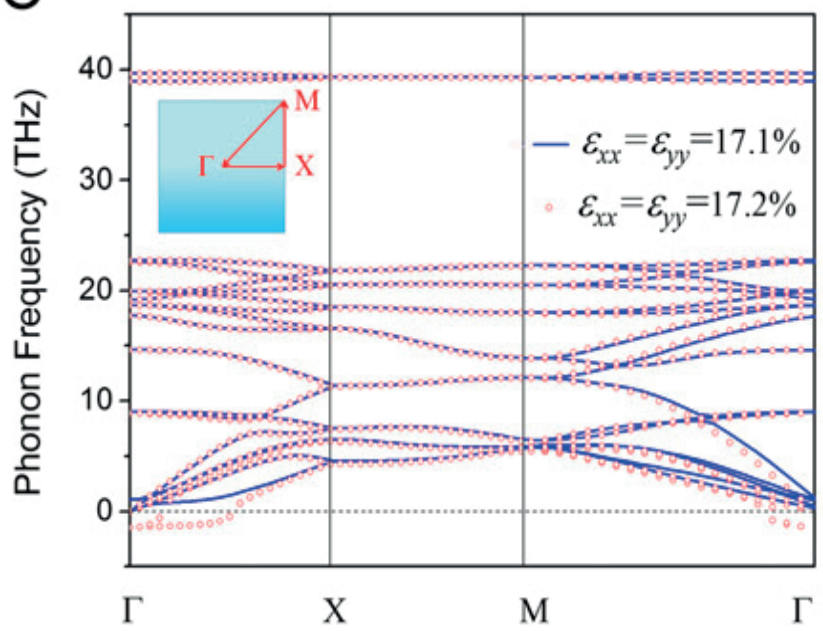

direction. We examine cases with $\varepsilon_{\mathrm{xx}}=5 \%, 6 \%$, and $7 \%$. As expected, we find that the equilibrium lattice constant in the $y$ direction is expanded in all of the cases (Fig.3A). This confirms the NPR of pentagraphene. It is well known that Poisson's ratio is defined as the negative ratio of the transverse strain to the corresponding axial strain. Normally, this ratio is positive as most solids expand in the transverse direction when subjected to a uniaxial compression. Although the continuum mechanics theory does not rule out the possibility of emergence of NPR in a stable linear elastic material, it is fairly rare to find such NPR material in nature. However, it has been found that some artificial materials have NPR and exhibit excellent mechanical properties [31,32]. Such materials, usually referred to as auxetic materials or mechanical metamaterials, are of broad interest in
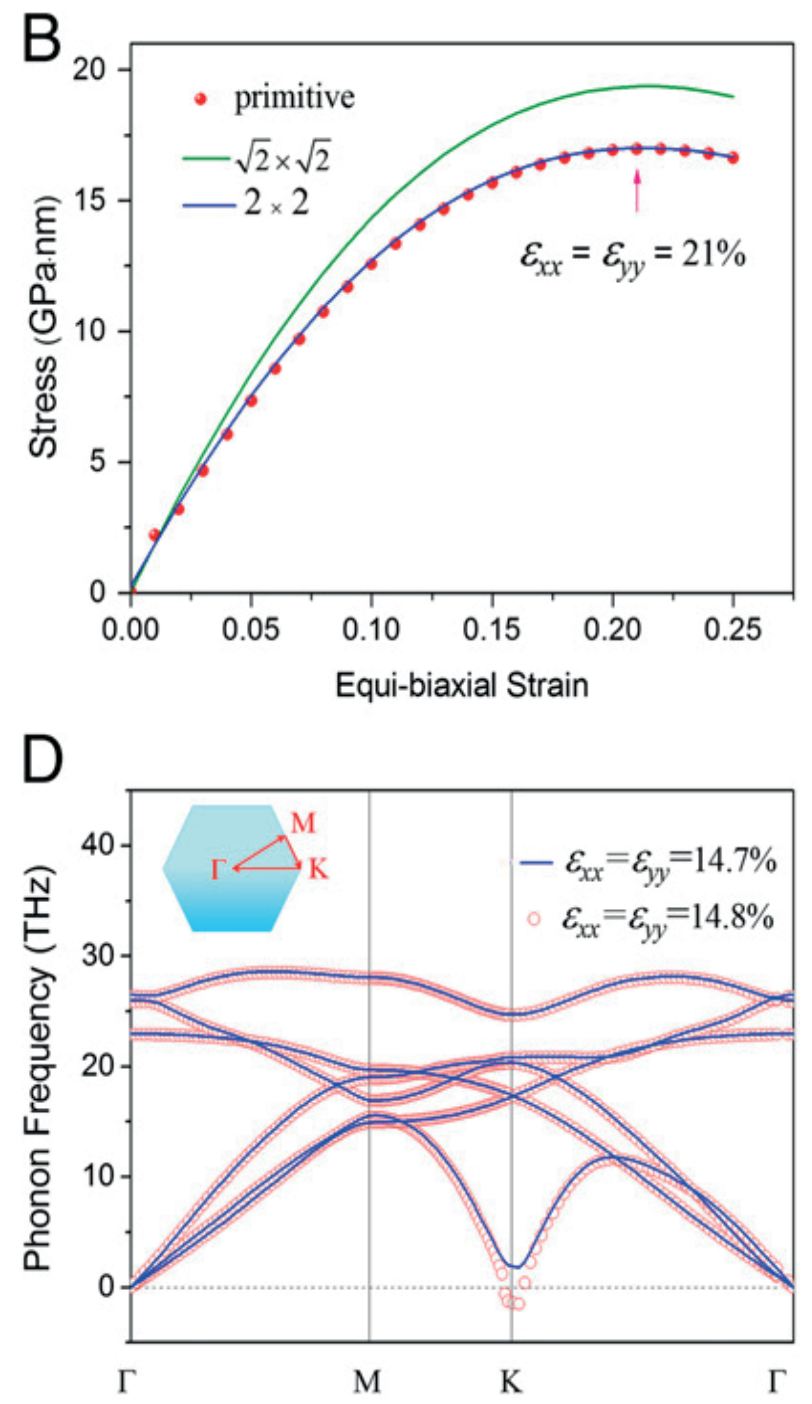

Fig. 3. (A) Strain energy with respect to the lateral lattice response when the penta-graphene lattice is under uniaxial strain along the $x$ direction. The arrows indicate the equilibrium magnitude of $\varepsilon_{y y}$. (B) Stress-strain relationship under equi-biaxial tensile strain. The red arrow denotes the maximum strain. (C) Phonon bands of penta-graphene at the extreme of equi-biaxial strain. (D) Same as C for graphene. Blue lines and red circles represent phonons before and after the failure, respectively. (Insets) The high-symmetry q-point paths in the reciprocal space. 
both scientific and technological communities [33]. Thus, penta-graphene with such unusual mechanical property may have multiple applications such as a tension activatable substrate, a nanoauxetic material, or a deformable variable-stiffness material.

Besides in-plane stiffness, ideal strength is also a very important mechanical property of a nanomaterial. We study the ideal strength of pentagraphene by calculating the variation of stress with equi-biaxial tensile strain using different cells. The results are plotted in Fig. 3B, which shows that the strain at the maximum stress is $21 \%$. Such an ultrahigh ideal strength is exciting. However, we should note that phonon instability might occur before mechanical failure. Such failure mechanism has been well studied in graphene where the phonon softening induced by Kohn anomaly occurs before the stress reaches its maximum in the primitive cell $[27,28]$. To check whether similar phonon-dominant failure mechanism exists in penta-graphene, we compute the phonons under increasing equi-biaxial strain. The results at the critical point of phonon softening are plotted in Fig. 3C. We find that phonon softening does not arise until the magnitude of equibiaxial strain reaches $17.2 \%$, which is smaller than the magnitude of $21 \%$ obtained from the stressstrain curve. For comparison, we also calculate phonons of the equi-biaxially stretched graphene. The observed softening of the $K_{1}$ mode at the Dirac point under equi-biaxial tensile strain of $14.8 \%$ (Fig. $3 \mathrm{D})$ is in excellent agreement with previous work [27, 28]. This indicates that the critical strain of pentagraphene is significantly larger than that of pristine

A

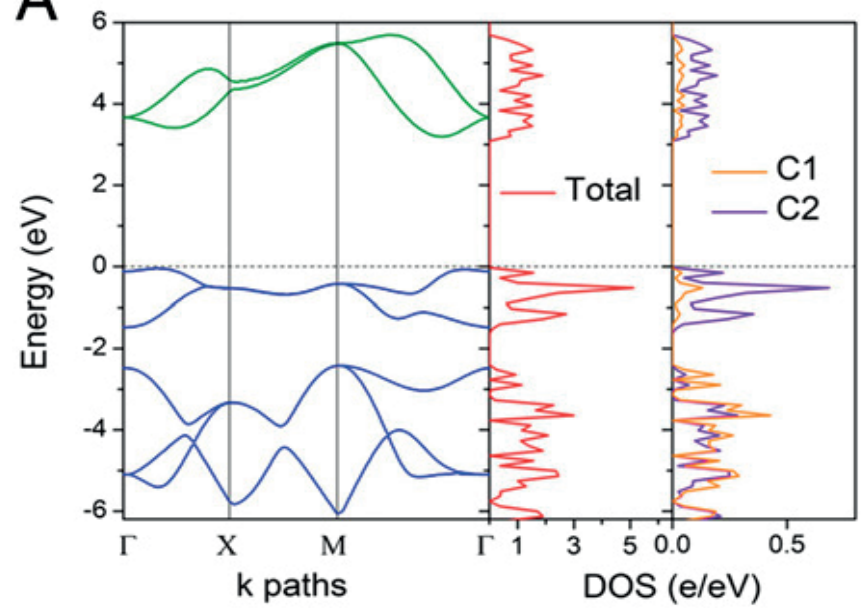

graphene. It is also comparable to that of carrierdoping-strengthened graphene [28]. At the critical strained state, the single bond lengths between $C 1$ and $C 2$ atoms reach $\sim 1.77 \AA$, which is comparable with the experimentally [34] and theoretically [35] reported longest $C-C$ bond length. Detailed analyses on the eigenvectors corresponding to the imaginary modes reveal that the structure fracture stems from the breakdown of some of the $\sigma$ bonds between $C 1$ and $C 2$ atoms.

2.7. Electronic Properties. To probe the electronic properties of penta-graphene, we calculate its band structure and corresponding total and partial density of states (DOS). As shown in Fig. 4A, pentagraphene is an indirect band-gap semiconductor with a band gap of $3.25 \mathrm{eV}$ [computed using the Heyd-Scuseria-Ernzerhof (HSE06) functional] [36, 37], because the valance band maximum (VBM) lies on the $\Gamma-X$ path whereas the conduction band minimum is located on the $M-\Gamma$ path. However, due to the existence of the sub-VBM on the $M-\Gamma$ path, which is very close to the true VBM in energy, pentagraphene can also be considered as a quasi-directband-gap semiconductor. Analysis of its partial DOS reveals that the electronic states near the Fermi level primarily originate from the $s p^{2}$ hybridized $C 2$ atoms, which is further confirmed by calculating the banddecomposed charge density distributions, as shown in Fig. 4B-E. A simplified tight-binding model is used to understand the underlying physics behind the band-gap opening feature in the band structure of penta-graphene (see SI Appendix, text S3 for details). We argue that it is the presence of the $s p^{3}$-hybridized
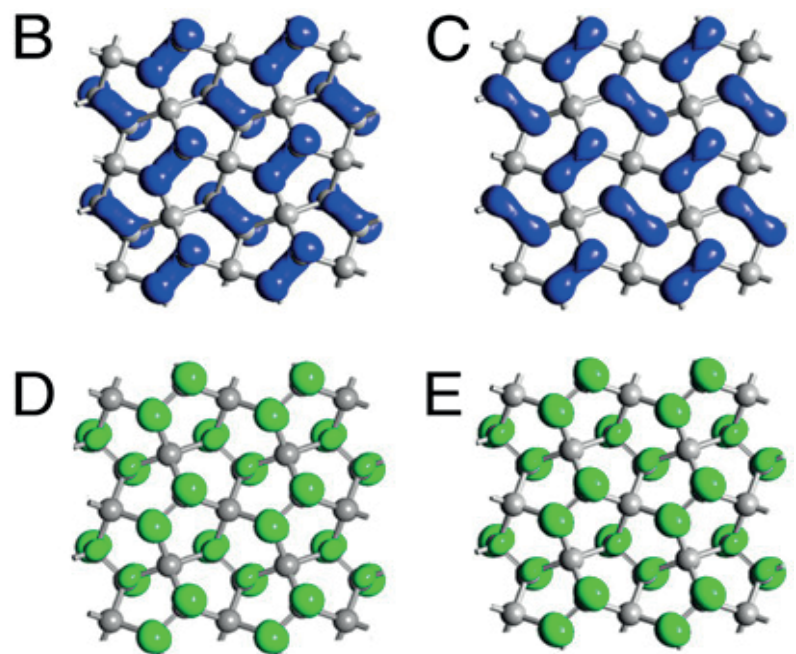

Fig. 4. (A) Electronic band structure and total and partial DOS of penta-graphene calculated by using HSE06 functional. The Fermi level is shifted to $0.00 \mathrm{eV}$. Band-decomposed charge density distributions are depicted in B to E: (B) the second highest occupied band, (C) the highest occupied band, (D) the lowest unoccupied band, and (E) the second lowest unoccupied band. 
C1 atoms that spatially separates the $p_{z}$ orbitals of $s p^{2}$-hybridized C2 atoms, hindering full electron delocalization and thus giving rise to a finite band gap. The dispersionless, partially degenerate valance bands lead to a high total DOS near the Fermi level, lending to the possibility that Bardeen-CooperSchrieffer superconductivity can be achieved in this nanosheet through hole doping [38].

\subsection{Penta-Tubes: Rolled-Up Penta-Graphene.} It is well known that the electronic properties of carbon nanotubes are closely related to graphene according to the zone folding approximation [39]. Due to the gapless semimetallic feature of graphene, the electronic properties of carbon nanotubes are highly chirality-dependent: a carbon nanotube is metallic only when its chiral vector $(n, m)$ satisfies $n-m=3 l$, where $l$ is an integer. The difficulty in fabricating and separating carbon nanotubes with certain conductance (metallic or semiconducting) greatly hinders its application in nanoelectronics. A previous study proposed a family of metallic carbon nanotubes based on metallic Heackelite sheet [11]. It is therefore natural to expect that the penta-graphene-based nanotubes could be semiconducting regardless of chirality. To test this hypothesis we have constructed a series of pentagon-based carbon nanotubes by rolling up the penta-graphene sheet along the $(n, m)$ chiral vectors, where $n=m$ range from 2 to 8 (Fig. 5A). The tubes with other chiralities $(n \neq m)$ failed to converge to stable tubular structures. We name these pentagonbased carbon nanotubes penta-tubes. The optimized geometry of a $(3,3)$ penta-tube is illustrated in Fig. 5B. The dynamic and thermal stability of this nanotube is confirmed by carrying out phonon calculations and AIMD simulations, respectively. The results are presented in Fig. 5C and SI Appendix, Fig. S7, respectively. We find that not only all of the $(n, n)$ penta-tubes are dynamically robust (SI Appendix, Fig. S8) but also they are thermally stable up to $1,000 \mathrm{~K}$. Analysis of their band structures and DOS reveals that all of the stable penta-tubes are semiconducting. The calculated results are summarized in SI Appendix, Table S2. Except for the highly curved $(2,2)$ penta-tube, the band gaps of the $(n, n)$ penta-tubes are not sensitive to their diameters. Thus, chirality-independent semiconducting carbon nanotubes can be produced for application in nanoelectronics. The semiconducting behavior of penta-tubes can be attributed to the electronic structure of penta-graphene, which resembles the case of other semiconducting monolayers such as $h-B N$ monolayer and the corresponding nanotubes [40] which inherit the semiconducting feature.

\subsection{D Carbon Structures: Stacked Penta-}

Graphene Layers. To further explore the structural versatility of penta-graphene, we have altered the stacking patterns of the penta-graphene layers,
A

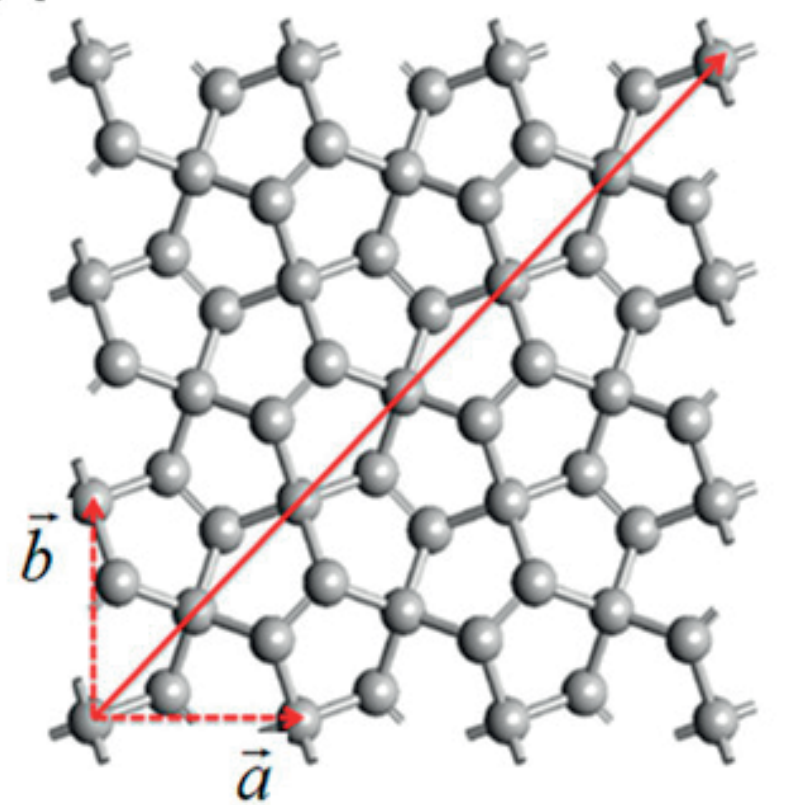

B

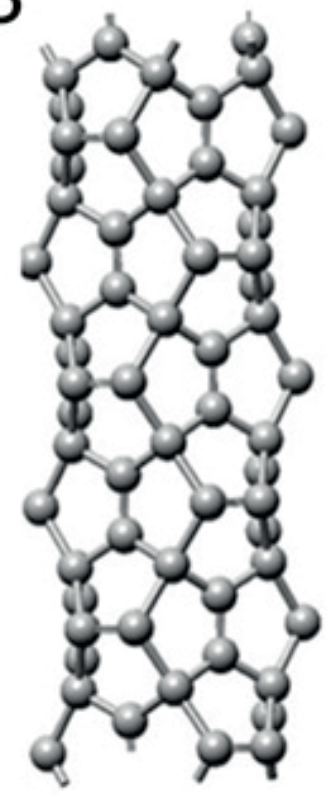

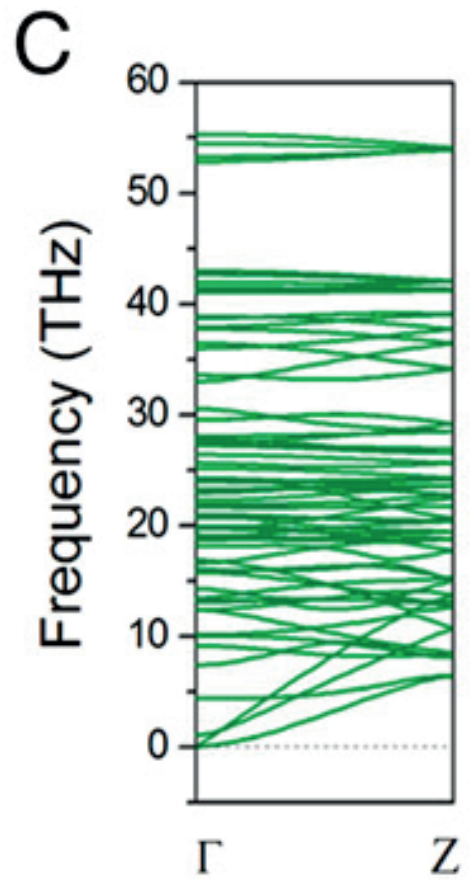

Fig. 5. (A) Illustration of chiral vectors of penta-tube. Dashed lines with arrows denote the lattice basis vector. (B) Optimized structure of $(3,3)$ penta-tube from side view, and $(C)$ the corresponding phonon spectra. 
leading to a $3 D$ structure as shown in Fig. $6 A$ and $B$. Following the nomenclature used to analyze the structural character of fused-pentagon-based Pentaheptite [14], we define the layer stacking in T12-carbon as $A B$ type. The stacking of the designed structure shown in Fig. $6 A$ and $B$ is then termed as $A A$ type. It can be viewed as a twin structure of the T12-carbon phase. The calculated phonon spectra of $A A-T 12$ are presented in Fig. $6 C$, confirming its dynamical stability. Indeed, more complicated structures are expected to be built, akin to the pentaheptite modification of graphene [14]. An example of such structures containing four penta-graphene layers (24 atoms) per unit cell, termed $A B A A-T 24$, is presented in SI Appendix, Fig. S9. The detailed structural information of these new $3 D$ phases as well as T12-carbon, for comparison, is given in SI Appendix, Table S3. The calculated cohesive energies (averaged on each carbon atom) are -8.87 , -8.92, and $-8.98 \mathrm{eV}$ for $A A-T 12, A B A A-T 24$, and T12-carbon, respectively, indicating that the $A A$ T12 and $A B A A-T 24$ phases are nearly as stable as T12-carbon. Band structure calculations suggest that both $A A-T 12$ and $A B A A-T 24$ are semiconducting with the energy band gaps of 5.68 and $5.33 \mathrm{eV}$, respectively, which are even larger than that of T12carbon $(4.56 \mathrm{eV})$, indicating that these phases can be highly electrically resistant and optically transparent like those products of cold compressed graphite [41]. The bulk moduli of these polymorphs are calculated

A

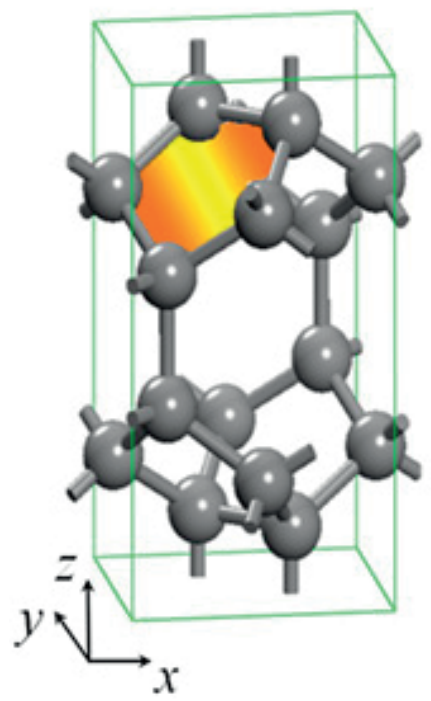

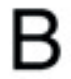

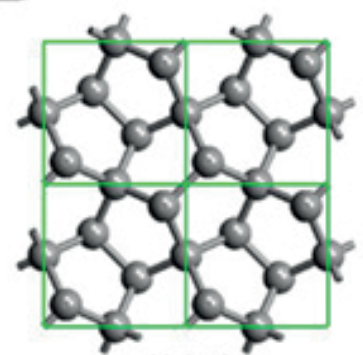

(001)

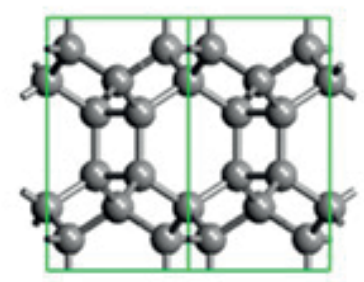

(100) by fitting the third-order Birch Murnaghan equation of states [42]. Although the bulk moduli of $A A-T 12$ (359 $\mathrm{GPa})$ and $A B A A-T 24(380 \mathrm{GPa})$ are slightly smaller than that of T12-carbon (403 GPa), they are comparable with that of cubic $B N$, suggesting their potential applications in machining. We note that a very recent theoretical work identified the $A A-T 12$ structure by considering its $S i$ analog [43]. It is also pointed out that, like T12-carbon, $A A-T 12$ is a universal structure shared by $C, S i, G e$, and $S n$, suggesting this family of tetragonal structures may be ubiquitous in elemental allotropes of group IVA elements.

\section{DISCUSSION}

We have demonstrated via AIMD simulations that the metastable penta-graphene structure can withstand very high temperature. We note that the experimentally synthesized dodecahedral $C_{20}$, the smallest carbon fullerene consisting of only carbon pentagons, is metastable, but possesses outstanding thermal stability (up to 3,000 K) [44]. These results imply that thermodynamic criteria may not be the deterministic factor in the synthesis of new carbonbased materials. In fact, due to the bonding versatility of carbon, the PES might be fairly complicated with numerous local minima (corresponding to metastable phases) separated from each other by considerable energy barriers. Graphite and cubic diamond are energetically superior to almost all other carbon polymorphs, namely, they correspond

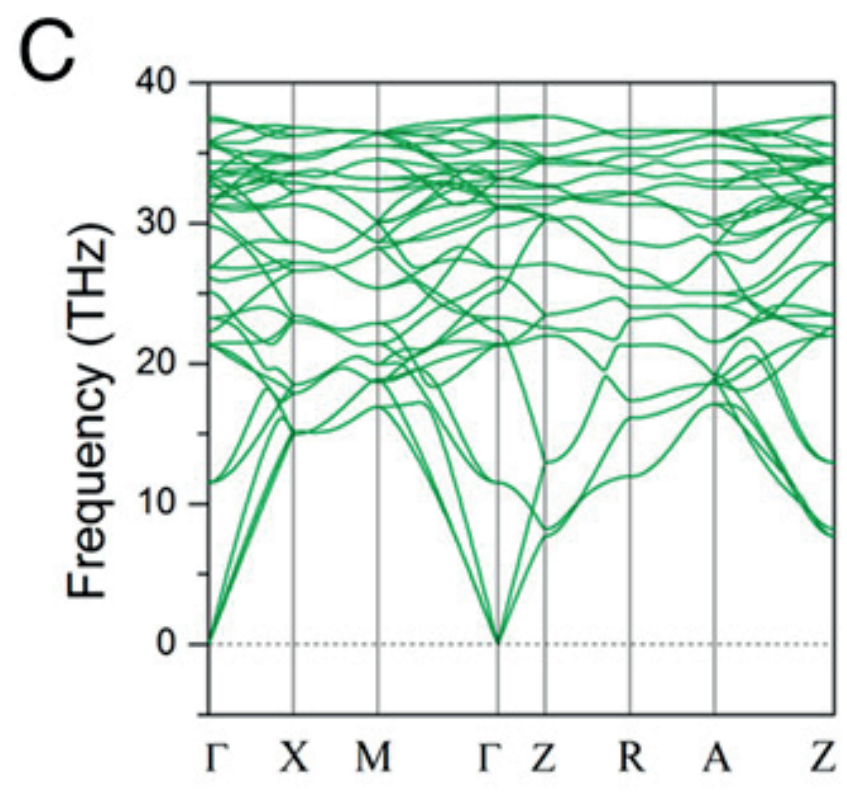

Fig. 6. (A) Crystal structure of AA-T12 carbon. (B) AA-T12 viewed from the [001] and [100] directions, and (C) the corresponding phonon spectra. 
to the two lowest-lying valleys on the PES. The high-energy barrier between graphite and diamond makes it possible for both graphite and diamond to coexist in nature. However, recent experimental [41] and theoretical [45] advances have identified many intermediate phases between graphite and diamond during cold compression of graphite. Some of these phases are pressure-recovered, i.e., they can exist when the external pressure is removed. This stability is also ascribed to the considerable energy barrier. In fact, even in some surfaces of carbon structures, different structural reconstruction patterns are separated by appreciable kinetic energy barriers [35]. These findings highlight the vital role that kinetics $[22,23]$ plays in carbon structure evolution, and fuel the exploration of new metastable carbon phases as functional materials.

We now reflect on the relationship between the special atomic configuration and exotic mechanical properties of penta-graphene. When the structure is under uniaxial tension, the expansive lateral response has two kinds of impact on the total energy: on one hand, it elongates the bond and weakens the binding energy; on the other hand, it significantly reduces the difference between the two lattice constants $a$ and $b$, thus helping the structure to get close to its original tetragonal symmetry, reducing the bond distortion around the $s p^{3}$-bonded $C 1$ atoms and lowering the strain energy. The structure thus evolves to its equilibrium as a result of compromise between these two competing factors. Detailed illustrations of atom evolution under uniaxial lattice stretch are presented in SI Appendix, text S4. Such regime is reminiscent of an earlier work [46] which argued that the combination of "chemical criteria" and "crystallographic criteria" in carbon materials can lead to exceptional mechanical properties. The ultrahigh critical strain of penta-graphene is also intimately related to its atomic structure. The buckled structure slows down the bond elongation and hence the structure is highly stretchable. Besides, graphene has topologically protected pointlike Fermi surface, and the coupling between the electron states near the Fermi level and certain phonon mode leads to fast phonon softening under biaxial tension [27], whereas penta-graphene does not suffer from Kohn anomaly, because it is semiconducting.

One practical issue in the synthesis of pentagraphene is how to selectively break the interlayer covalent bonds in T12-carbon [18]. To address this challenge, we point to a similar strategy where hydrogen intercalation was successfully used to decouple a graphene layer from the H-SiC (0001) surface $[47,48]$. Details of our exfoliation scheme are given in SI Appendix, text $S 5$.

In summary, we showed that a $2 \mathrm{D}$ carbon sheet, penta-graphene, composed entirely of pentagons can be obtained by chemically exfoliating a single layer from the T12-carbon phase. Although pentagraphene is energetically metastable compared with graphene, it is dynamically stable and can withstand temperatures up to 1,000 K. Due to its special atomic configuration, penta-graphene has unusual properties, such as (i) it exhibits NPR, similar to that recently reported in a single-layer black phosphorus sheet [32]; (iv) it exhibits ultrahigh ideal strength that can even outperform graphene; (iii) it is semiconducting, thus, there is no need to functionalize penta-graphene for opening the band gap as is the case with graphene. In addition, pentagraphene can be rolled up to form a $1 D$ pentagonbased nanotube that is semiconducting regardless of its chirality. Therefore, there is no need to develop special techniques to separate semiconducting nanotubes from the metallic ones as is the case with conventional carbon nanotubes. Penta-graphene can also be stacked to form $3 D$ stable structures displaying different properties from those of the mother-phase T12-carbon. Thus, penta-graphene sheet not only possesses exotic properties by itself but also can be used to build new structures. We hope that these findings will motivate experimental efforts. Once synthesized, these new carbon allotropes may not only enrich carbon science but also may lead to an untold number of applications.

\section{METHODS}

First-principles calculations and AIMD simulations within the framework of density functional theory are performed using Vienna $\mathrm{Ab}$ initio Simulation Package (VASP) [49]. The 2D system is separated from its periodic images by a vacuum distance of $20 \AA$ in the perpendicular direction. Projector augmented wave (PAW) [50] method is used to treat interactions between ion cores and valance electrons. Plane waves with a kinetic energy cutoff of $500 \mathrm{eV}$ are used to expand the valance electron $\left(2 s^{2} 2 p^{2}\right)$ wavefunctions. The exchange-correlation 
potential is incorporated by using the generalized gradient approximation [51] due to Perdew-BurkeErnzerhof in most of our calculations whereas a hybrid HSE06 [36, 37] functional is used for highaccuracy electronic structure calculations. The first Brillouin zone is represented by $K$ points sampled using the Monkhorst-Pack scheme [52] with a grid density of $2 \pi \times 0.02 \AA^{-1}$. For geometry relaxation, the convergence thresholds for total energy and atomic force components are set at $10^{-4} \mathrm{eV}$ and $10^{-3} \mathrm{eV} \AA^{-1}$, respectively. In AIMD simulations the convergence criterion of total energy is set as $1 \mathrm{meV}$. Temperature control is achieved by Nosé thermostat [53]. Structure relaxations are performed without any symmetry constraint. Phonon properties are calculated using finite displacement method implemented in Phonopy [54]. A (4×4) supercell is constructed to calculate the atomic forces by using VASP, with a very high accuracy (stringent energy convergence criteria $10^{-8} \mathrm{eV}$ per unit cell). Phonon calculations using a larger supercell $(6 \times 6)$ yield consistent results. For phonon calculations in graphene, an $(8 \times 8)$ supercell is used, which has been found in previous work [27] to be sufficient to take into account the long-range interatomic interactions.

\section{SI APPENDIX}

\section{SI TEXT}

Text S1. Comparison of the ZA branch of pentagraphene and graphene

To quantitatively compare the ZA branch of graphene and penta-graphene, we first fit the long-wave relationship $\omega=c q^{2}$ (data points with a frequency lower than $80 \mathrm{~cm}^{-1}$ are used for fitting) to obtain the coefficient $c$. We fit the coefficient $c$ of penta-graphene along the $\Gamma-X$ and $\Gamma-M$ directions, which are found to be $6.08 \times 10^{-7} \mathrm{~m}^{2} \mathrm{~s}^{-1}$ and $5.82 \times 10^{-7} \mathrm{~m}^{2} \mathrm{~s}^{-1}$, respectively, exhibiting a slight anisotropy due to its tetragonal symmetry. The fitted $c$ value for graphene is $5.40 \times 10^{-7} \mathrm{~m}^{2} \mathrm{~s}^{-1}$, which is in good agreement with previous result [1].

Quantitatively, the frequency-wave vector relationship, in the vicinity of the $\Gamma$ point, can be formulated as: $\omega=c q^{2}=\sqrt{D / \rho} q^{2}$ [2], where $D$ and Q correspond to the bending modulus and the mass area density of the nanosheet, respectively. We obtain the bending stiffness of graphene to be 1.36 $\mathrm{eV}$, which is in good agreement with previous results $[1,3]$. Unlike graphene which has isotropic in-plane

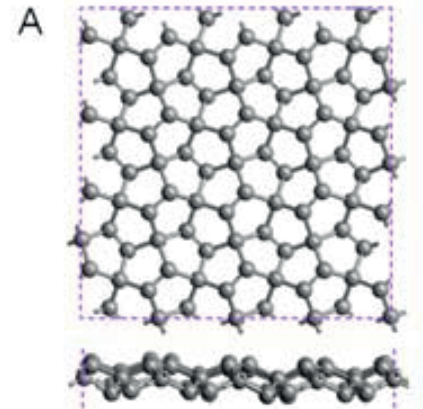

$4 \times 4,300 \mathrm{~K}$

C

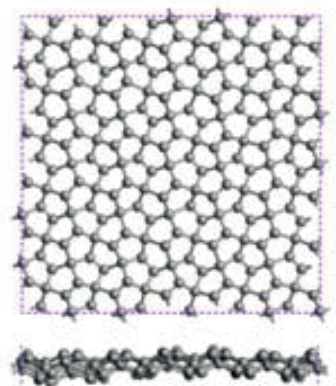

$6 \times 6,1000 \mathrm{~K}$
B

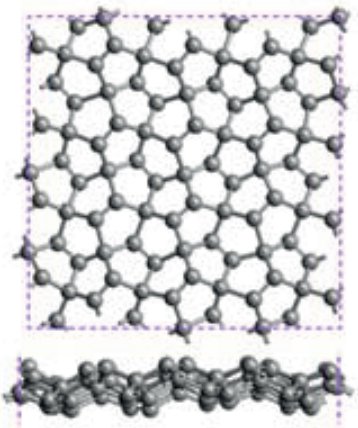

$4 \times 4,1000 \mathrm{~K}$

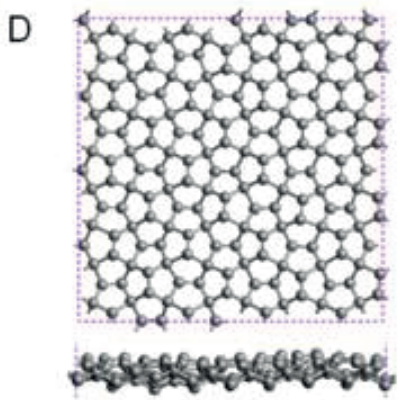

$4 \sqrt{ } 2 \times 4 \sqrt{ } 2,1000 \mathrm{~K}$
Fig. S1. Snapshots of atomic configurations of penta-graphene at the end of AIMD simulations. The simulated supercells are marked by purple squares, and their corresponding size and temperature are denoted below the each panel.

mechanical behavior due to the hexagonal symmetry, the $Z A$ branch of penta-graphene shows directiondependent dispersion and hence the bending rigidity is anisotropic. Our estimated bending moduli of penta-graphene along the axial and diagonal direction are 1.90 and $2.08 \mathrm{eV}$, respectively.

The ZA mode is also argued to be closely related to thermodynamic properties as it has the lowest vibrational energy among all modes and is most likely to be activated at low temperature.

Given the relationship $\omega=c q^{2}$ for the $Z A$ mode, vibration density of states can be written as, $g(\omega)=\frac{S}{(2 \pi)^{2}} \int \frac{d l}{\left|\nabla_{q} \omega(q)\right|}=\frac{S}{(2 \pi)^{2}} \frac{2 \pi q}{2 c q}=\frac{S}{4 \pi c}$,

where $S$ is the unit cell area. The Debye frequency $\omega_{\mathrm{D}}$ can be determined by $N=\int_{0}^{\omega_{D}} g(\omega) d \omega=\frac{S \omega_{D}}{4 \pi c}$.

Here $N$ is the number of unit cells, and $\omega_{D}$ is the Debye frequency. In this case, the energy related to temperature can be written as

$$
\begin{aligned}
& E(T)=\int_{0}^{\omega_{D}} g(\omega) \frac{\hbar \omega}{e^{\hbar \omega / k_{B} T}-1} d \omega=\frac{S}{4 \pi c} \int_{0}^{\omega_{D}} k_{B} T \frac{\hbar \omega / k_{B} T}{e^{\hbar \omega / k_{B} T}-1} d \omega= \\
& =\frac{N\left(k_{B} T\right)^{2}}{k_{B} \theta_{D}} \int_{0}^{\frac{\theta_{D}}{T}} \frac{x}{e^{x}-1} d x=\frac{N k_{B} T^{2}}{\theta_{D}} \int_{0}^{\frac{\theta_{D}}{T}} \frac{x}{e^{x}-1} d x,
\end{aligned}
$$


here $\theta_{D}=\hbar \omega_{D} / k_{B}$ and $x=\hbar \omega / k_{B} T$.

The heat capacity contributed by the $Z A$ mode can then be written as,

$$
C_{V}^{Z A}(T)=\frac{\partial E}{\partial T}=\frac{\partial}{\partial T}\left(\frac{N k_{B} T^{2}}{\theta_{D}} \int_{0}^{\frac{\theta_{D}}{T}} \frac{x}{e^{x}-1} d x\right) .
$$

At the low temperature limit, the integral goes from 0 to infinity. Considering

$$
\int_{0}^{+\infty} \frac{x}{e^{x}-1} d x=\frac{\pi^{2}}{6}
$$

we obtain

$$
C_{V}^{Z A}(T)=\frac{N \pi^{2} k_{B}}{3} \frac{T}{\theta_{D}}=\frac{\pi k_{B}^{2} S}{12 \hbar c} T, \quad T \rightarrow 0 .
$$

Here we see that the $Z A$ mode contributed heat capacity $C_{V}{ }_{V} A \sim T /$ cat low temperature. The calculated unit cell area for graphene and penta-graphene are $5.27 \AA^{2}$ and $13.25 \AA^{2}$, respectively, and with the fitted $c$ values in hand, the ratio of $C_{V}^{Z A}$ (penta-graphene)/ $C^{V}{ }_{Z A}$ (graphene) is calculated to be 2.3. This indicates that the $Z A$ branch of penta-graphene contributes more to the heat capacity as compared to that of graphene.

Text S2. Stability of penta-graphene in the presence of defects

The defected penta-graphene is simulated by using a $4 \times 4$ supercell containing 96 atoms. The studied defects include monovacancies at the 4- and 3 -coordinated $C$ sites, respectively, the divacancy as missing a pair of the 3-coordinated C2 atoms, the

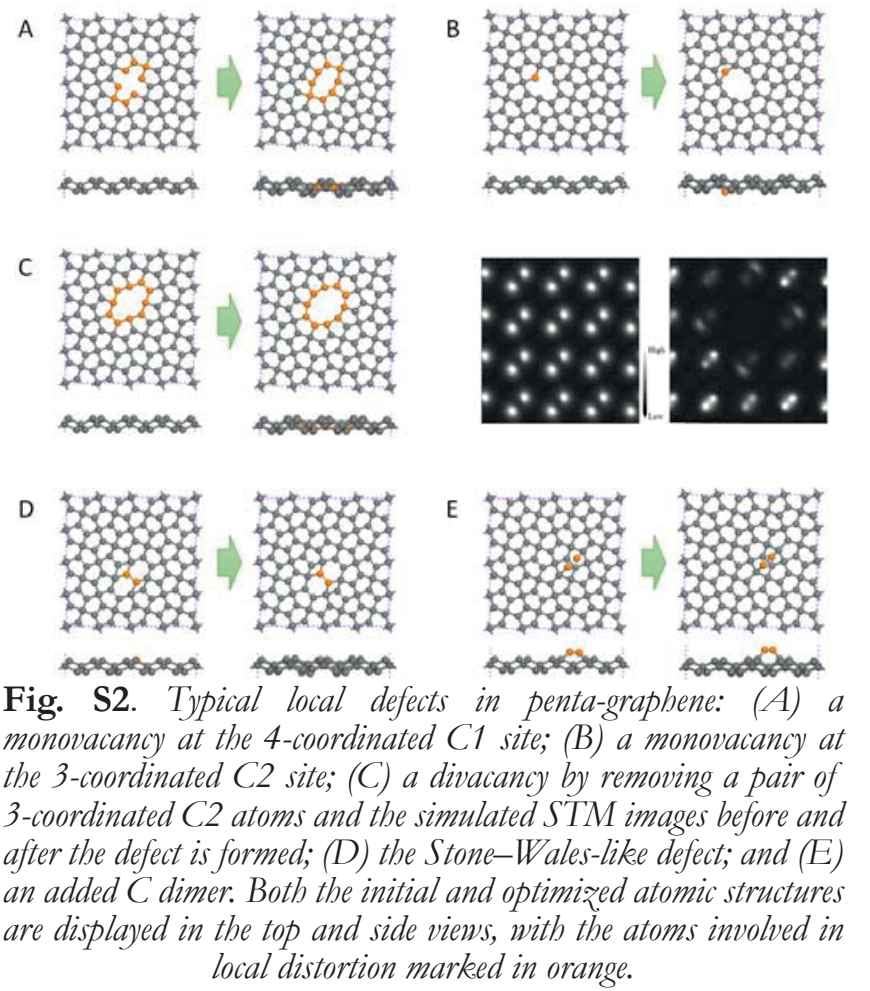

Stone-Wales-like defect by rotating a pair of the 3 -coordinated $C 2$ atoms with an angle of 90 degrees, and the adatom by adding a $C$ dimer at the two $C 2$ sites crossing above another pair of $C 2$ atoms, as shown in Fig. S2. The simulated STM images (under $-1.0 \mathrm{~V}$ bias voltages) for the perfect as well as the divacancy defected penta-graphene are presented in Fig. S2C. We note that in such a supercell, defect concentration reaches $4.7 \times 10^{17} \mathrm{~cm}^{-2}$, significantly exceeding the experimentally observed vacancy concentration $\left(\sim 3 \times 10^{11} \mathrm{~cm}^{-2}\right)$ in graphene [4]. It is found, after full geometry optimization, that atomic structures of all the defected penta-graphenes are well sustained with almost the same buckling height (see the side views in Fig. S2). Ab initio molecular dynamics simulations at $300 \mathrm{~K}$ are also carried out to confirm their thermal stability. The simulated results are given in Fig. S3 which shows that the integrity of the defected structures is still maintained after the systems are heated for five picoseconds ( $p s)$. No Domino effect appears and the total potential energies remain almost invariant. Therefore, the effect of the defects on the geometry of pentagraphene is "local". The structural stability as well as the thermal stability of the defected penta-graphene sheet suggests that penta-graphene is robust against defects as they can either partially release the stress or form more $s p^{3}$ hybridized $C$ atoms.

The effect of edge atoms on the structural stability of penta-graphene is also considered by studying a penta-graphene nanoribbon. Our AIMD
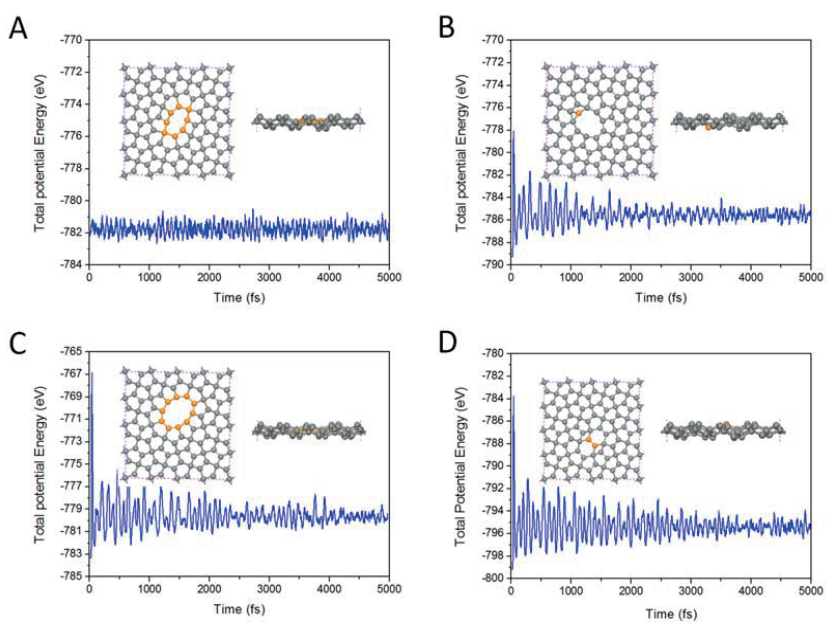

Fig. S3. Total potential energy fluctuation during AIMD simulations of penta-graphene with the defects: (A) a monovacancy at the $C 1$ site; (B) a monovacancy at the $C 2$ site; (C) a divacancy by removing a pair of $C 2$ atoms; (D) the Stone-Wales-like defect. The insets show the snapshot at 5 ps for each simulation. 


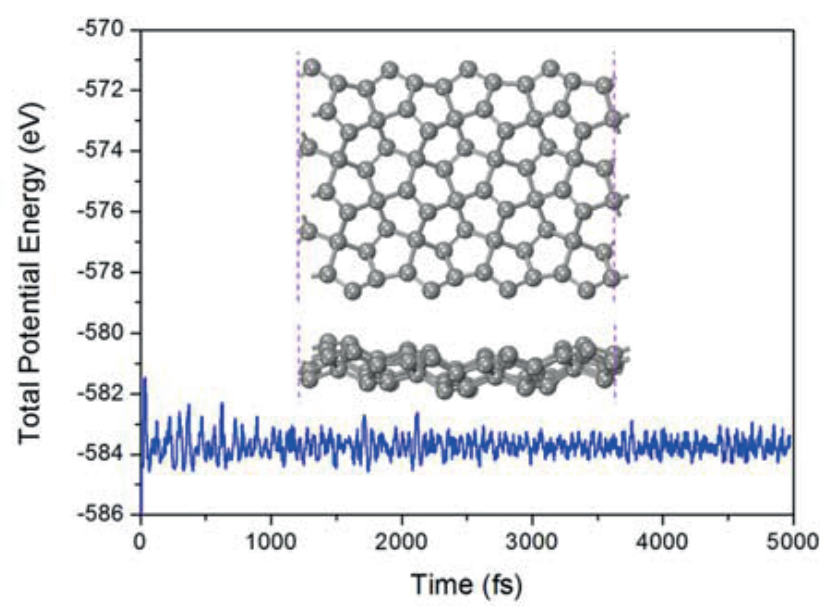

Fig. S4. Total potential energy fluctuation of a penta-graphene nanoribbon during AIMD simulations at $300 \mathrm{~K}$. The inset shows the snapshot at the end of simulation, with purple dashed lines denoting the unit cell in the periodic direction.

simulations (Fig. S4) indicate that the nanoribbon can withstand mild thermal perturbation at room temperature, which may be important for the synthesis.

Text S3. Discussion on the origin of finite band gap in penta-graphene

In graphene, all $C$ atoms are $s p^{2}$-hybridized, thus each atom has an intact $p_{z}$ orbital. These parallelly aligned $p_{z}$ orbitals form delocalized states throughout the whole sheet, leading to two dispersive $\pi$ and $\pi^{*}$ bands crossing at the Fermi level. The interactions of $p_{z}$ orbitals, which is responsible for the gapless feature of graphene, can be described by a simple tight-binding Hamiltonian $H=-t \sum\left(\hat{c}_{i}^{\dagger} \hat{c}_{j}+H . c.\right)$, with a constant hopping integral $t$. Introduction of $s p^{3}$-hybridized $C$ atoms destroys the homogenously

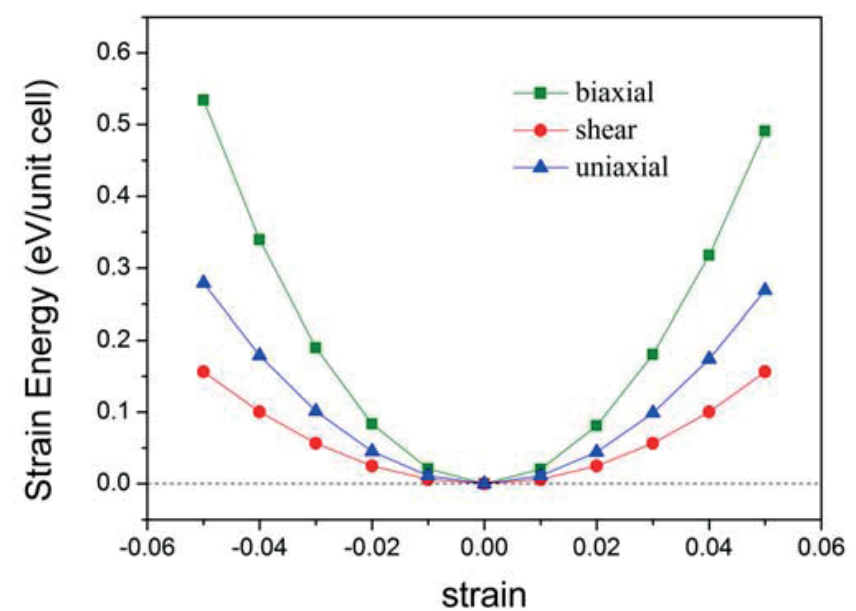

Fig. S5. Strain energy of penta-graphene under different kinds of in-plane strain. Note that the uniaxial strain curve is calculated by intentionally fixing the lateral lattice constant so that the strain energy can be simplified into the form $U(\varepsilon)=(1 / 2) C_{11} \varepsilon_{x x}{ }^{2}$.
A
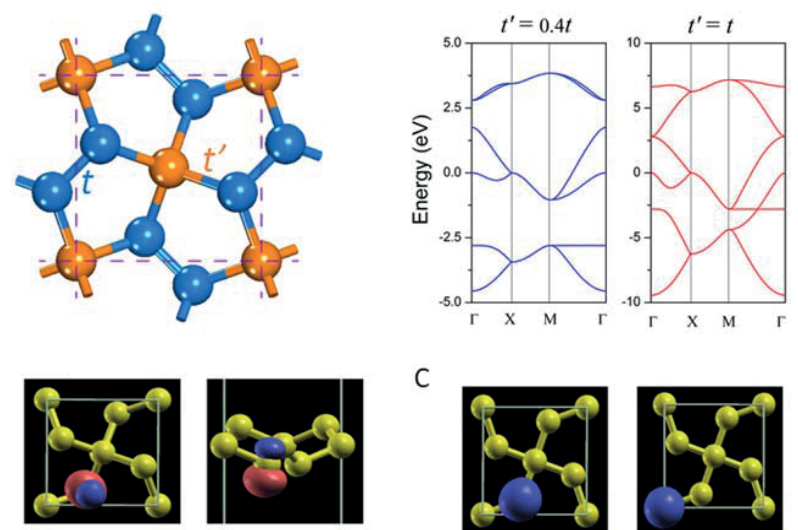

C
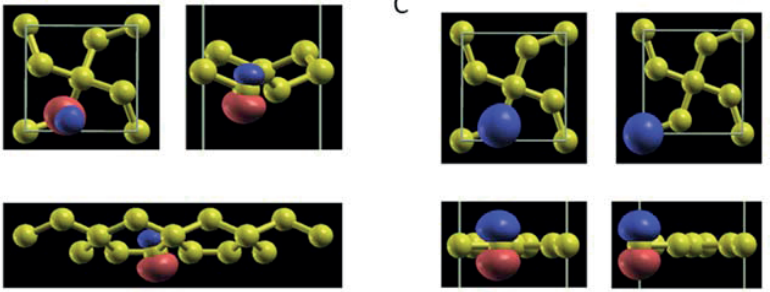

Fig. S6. (A) Illustration of hopping in the tight binding model and the corresponding bands with $t^{\prime}=0.4 t$ and $t^{\prime}=t$. (B) Contours of MLWFs of penta-graphene with the C2-2p, characteristics. (C) Contours of MLWFs of flat penta-graphene with the $2 p_{z}$ characteristics.

delocalized $\pi$ and $\pi^{*}$ states and opens a band gap, as seen in graphane [5].

Our computed orbital-resolved PDOS and band decomposed charge density show that the frontier bands of penta-graphene are dominated by the $p_{z}$ states of $s p^{2}$-hybridized $C 2$ atoms. However, these orbitals are spatially separated by the $s p^{3}$-hybridized C1 atoms and full delocalization is hindered, resembling the case of graphane. We construct a modified tight-binding Hamiltonian to describe the interaction of these orbitals (Fig. S6A):

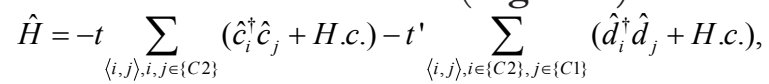

where the parameter $t$ denotes the hopping integral between the two C2- $p_{z}$ orbital, $t$ ' is the hopping

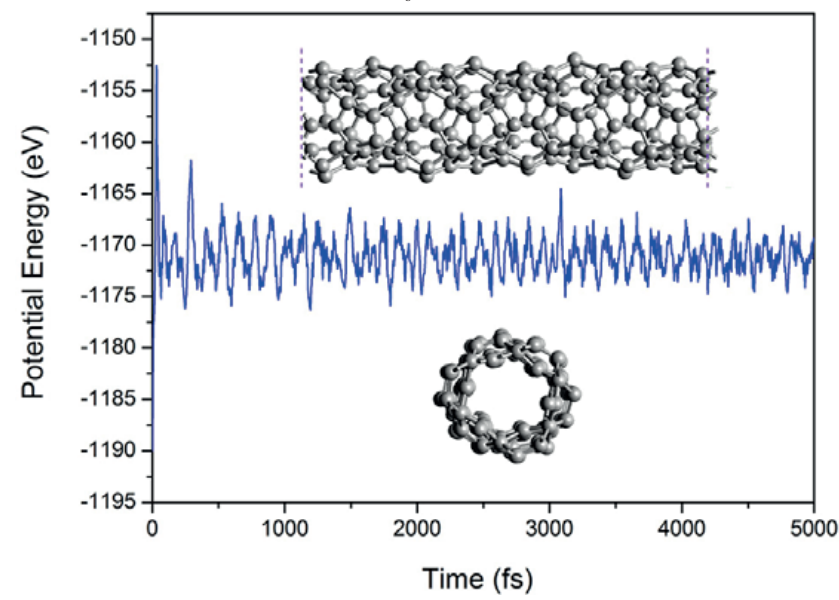

Fig. S7. Evolution of potential energy of $(3,3)$ penta-tube during AIMD simulations at $1000 \mathrm{~K} .1 \times 1 \times 4$ supercell is constructed for simulation to reduce the constraint of periodic condition in the axial direction. The insets show snapshots of atomic configuration at the end of the simulation. 


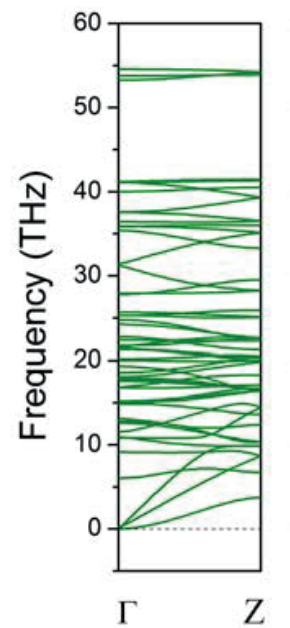

$(2,2)$

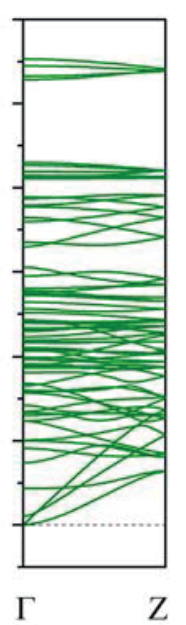

$(3,3)$

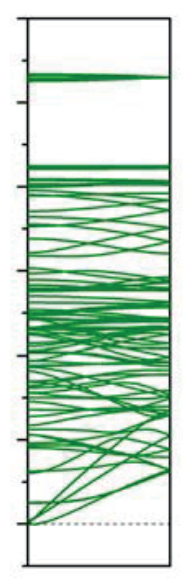

$(4,4)$

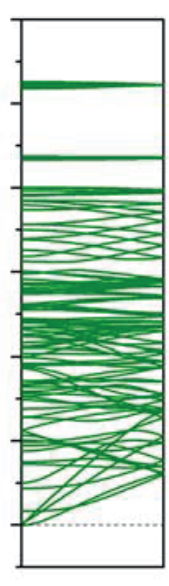

$(5,5)$

Fig. S8. Phonon spectra of penta-graphene-based nanotubes in different sizes: The high symmetric $q$ point path is along the $\Gamma$ $(0,0,0) \rightarrow Z(0,0,1 / 2)$ corresponding to the axial direction of nanotubes in the real space. The chiral vector number of each nanotube is denoted below the corresponding phonon spectra. integral between a C2- $p_{z}$ orbital and a C1-sp $p^{3}$ orbital. $c_{i}^{\dagger}\left(d_{i}^{\prime}\right)$ is the creation operator on the site $i$, and $c_{j}\left(d_{j}\right)$ is the corresponding annihilation operator at site $j$.

We alter the $t^{\prime} / t$ ratio to examine the band evolution. Two typical cases are observed: when $t^{\prime}=0.4 t$, the gapped feature is observed in tight binding bands and the basic features of the DFT bands are qualitatively reproduced, whereas setting $t^{\prime}=t$ leads to closing of the band gap (Fig. S6A). The former case can be easily understood as the overlap between two $C 2-p$, orbitals is obviously larger than that between a $C 2-p_{z}$ orbital and a $C 1-s p^{3}$ orbital, considering their orientations. The calculated

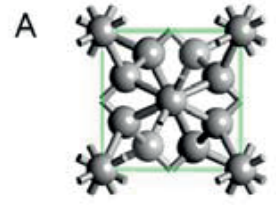

(001)

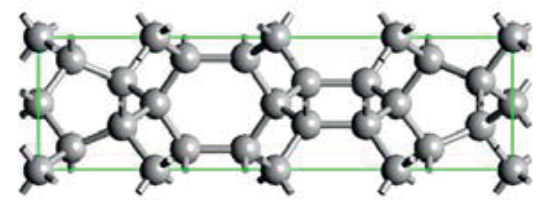

(100)

B

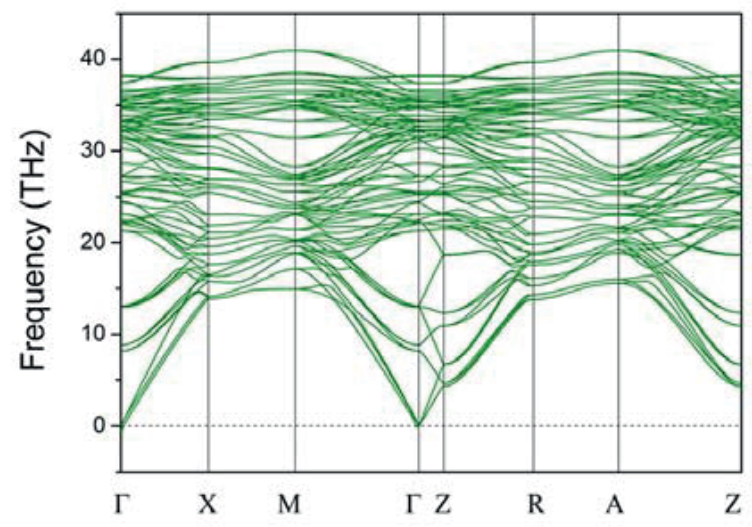

Fig. S9. $3 D A B A A-T 24$ carbon phase derived from Pentagraphene. (A) Crystal structure and (B) phonon spectrum.

maximally localized Wannier Functions (MLWF) [6, 7] of penta-graphene projected onto certain atomic orbitals (Fig. S6B) shows an "asymmetric lobe" feature indicating that these orbitals are perturbed due to the imperfect $s p^{2}$ hybridization. To understand the underlying physics of the band gap closing in the $t^{\prime}=t$ case, we need to alter the penta-graphene structure to enhance the C1-C2 hopping, to this end a hypothetical "flat penta-graphene (FPG)" model is constructed by compressing penta-graphene into a planar sheet. In this circumstance each planar tetracoordinated $C 1$ atom possesses an unhybridized $p_{z}$ orbital [8], which is also confirmed by our calculated MLWFs (Fig. S6C). DFT calculation indicates FPG is metallic, consistent with the tight binding result with $t=t^{\prime}$.

Based on the above mentioned results and analyses, we can conclude that it is the existence of $s p^{3}$-hybridized $C 1$ atoms that hinders the full delocalization of $C 2 p_{z}$ orbitals, and the resultant localized states lead to less dispersive bands with a gap near the Fermi level.

Text S4. Illustration of the origin of NPR effect in penta-graphene

To illustrate the origin of NPR effect in pentagraphene we begin with a simple model, namely that of a methane molecule with $T_{d}$ symmetry, plotted in the left panel of Fig. $\mathbf{S} 10 A$. The $\overline{4}$ axis as well as the two mirror planes $m 1$ and $m 2$ are also displayed. We performed simple DFT calculations to study how the

A
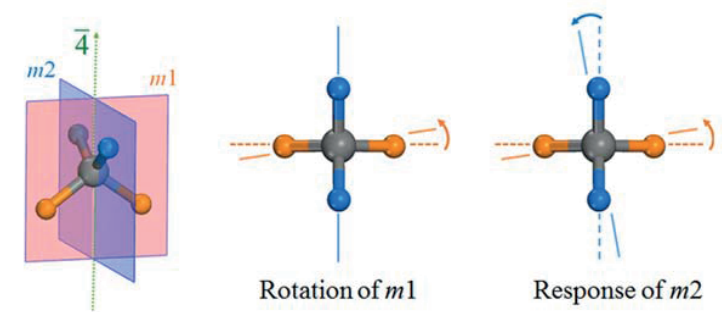

B
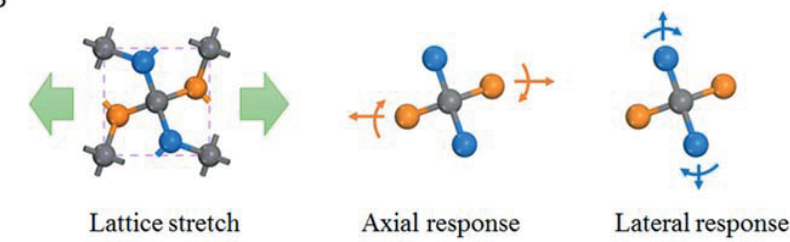

Fig. S10. Schematic illustration of the origin of auxetic behavior of penta-graphene. (A) The symmetric response in a $\mathrm{CH}_{4}$ molecule. (B) Schematic illustration of the response of $s p^{2}$-bybridized $C$ atoms in the penta-graphene unit cell when the lattice is uniaxially stretched. Arrows indicate the motion of atoms and rotation of the corresponding planes. 
A

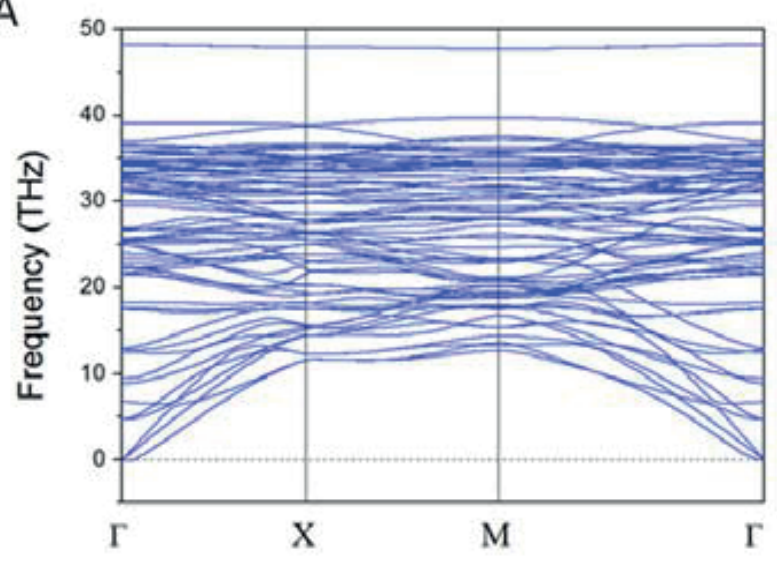

B

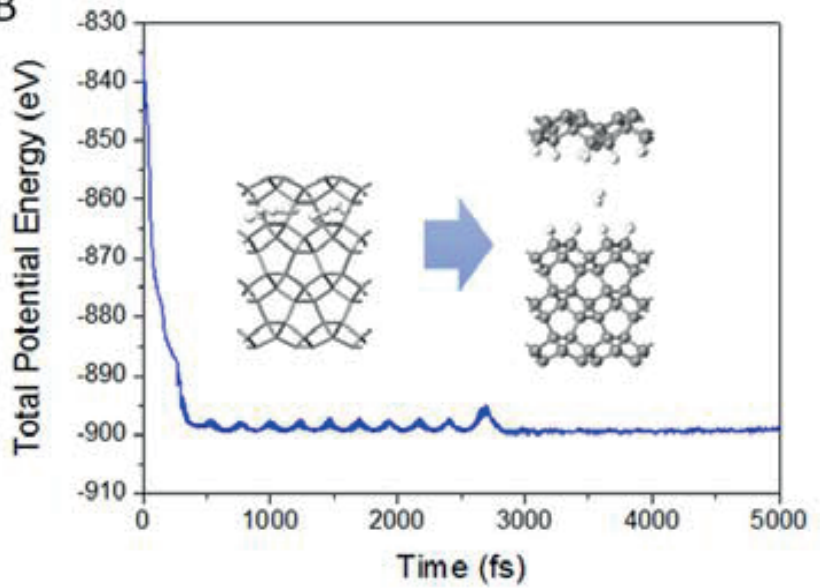

Fig. S11. (A) Phonon spectrum of a T12-carbon (001) thin film containing four penta-graphene layers. (B) Energy evolution of bydrogen intercalated T12-carbon thin film during AIMD simulations. The insets show the initial and final configurations.

$H$ atoms in the $m 2$ plane respond when the atoms in the $\mathrm{m} 1$ plane suffer a distortion. If the $m 1$ plane rotates along the $\overline{4}$ axis, $\mathrm{H}$ atoms in the $m 2$ plane rotate adaptively to minimize Coulomb repulsion and damp the system from deviation from the $T_{d}$ symmetry, as shown in the right panel of Fig. $\mathrm{S} 10 A$. In penta-graphene, when the lattice endures uniaxial tension, the $s p^{2}$-hybridized $C 2$ atoms evolve in a similar way. We assume that the central $s p^{3}$-hybridized $C 1$ atom is fixed. When stretching the lattice, the $C 2$ atoms, colored orange in Fig. $\mathrm{S} 10 B$, move outward along the tensile direction, leading to a clockwise rotation of the $m 1$ plane. Consequently, the $m 2$ plane rotates in the same direction, resulting in an expansive lateral response to the lattice. Therefore, NPR effect is exhibited in penta-graphene.

Text S5. A possible pathway to synthesize a monolayer penta-graphene

It has been demonstrated experimentally and theoretically that a graphene layer grown on $\mathrm{SiC}$ (0001) surface can be decoupled from the outermost Si layer by selectively breaking the covalent $S i-C$ bonds using hydrogen intercalation $[9,10]$. Following this idea, we have simulated the effect of hydrogen intercalation in T12-carbon for obtaining a pentagraphene sheet.

To mimic the chemical exfoliation procedure in T12-carbon, we first computationally cleaved a (001) thin film from T12-carbon and carefully confirmed its structural stability via phonon calculation (Fig. S11 $A$ ) and AIMD simulations. No surface reconstructions were found at $300 \mathrm{~K}$, implying that the film can serve as a suitable precursor for chemical exfoliation. At the initial point, all hydrogen atoms existed in molecular form with nearly-uniform distribution and random orientation with the minimum $\mathrm{C}-H$ distance larger than $1.5 \AA$. When the system reached new equilibrium, a penta-graphene monolayer with partial hydrogenation is separated and lifted from the thin film surface (Fig. S11B). This process is quite similar to what happened in graphene-SiC $[9,10]$. Our simulations indicate that hydrogen intercalation may be a viable way to selectively break the interlayer $C-C$ bonds in T12-carbon and to chemically exfoliate a penta-graphene monolayer. Penetration of hydrogen directly through the thin film surface may encounter high energy barriers like that in the case of graphene, but we note that such penetration may occur in the vicinity of local defects or grain boundaries [9]. In a realistic situation the topmost carbon layers may also be hydrogenated by exposing to hydrogen atmosphere which may help to prevent the quasi freestanding sheet from folding. Subsequent dehydrogenation of penta-graphene may be achieved through annealing approach demonstrated in Ref [5].

\section{REFERENCES TO TEXTS S1-S5}

1. Sánchez-Portal D, Artacho E, Soler JM, Rubio A, Ordejón P. Ab initio structural, elastic, and vibrational properties of carbon nanotubes. Phys Rev B, 1999, 59(19):12678-12688.

2. Muñoz E, Lu J, Yakobson BI. Ballistic Thermal Conductance of Graphene Ribbons. Nano Lett, 2010, 10(5):1652-1656.

3. Wei Y, Wang B, Wu J, Yang R, Dunn ML. Bending Rigidity and Gaussian Bending Stiffness of Single-Layered Graphene. Nano Lett, 2012, 13(1):26-30.

4. Ugeda MM, Brihuega I, Guinea F, Gómez-Rodríguez JM. Missing Atom as a Source of Carbon Magnetism. Phys Rev Lett, 2010, 104(9):096804.

5. Elias DC, et al. Control of Graphene's Properties by Reversible Hydrogenation: Evidence for Graphane. Science, 


\section{9, 323(5914):610-613.}

6. Mostofi AA, et al. Wannier90: A tool for obtaining maximally-localised Wannier functions. Comput Phys Commun, 2008, 178(9):685-699.

7. Marzari N, Mostofi AA, Yates JR, Souza I, Vanderbilt D. Maximally localized Wannier functions: Theory and applications. Rev Mod Phys, 2012, 84(4):1419-1475.

8. Hoffmann R, Alder RW, Wilcox CF. Planar tetracoordinate carbon. J Am Chem Soc, 1970, 92(16):4992-4993.

9. Riedl C, Coletti C, Iwasaki T, Zakharov AA, Starke U. Quasi-Free-Standing Epitaxial Graphene on SiC Obtained by Hydrogen Intercalation. Phys Rev Lett, 2009, 103(24):246804.

10. Sołtys J, Piechota J, Ptasinska M, Krukowski S. Hydrogen intercalation of single and multiple layer graphene synthesized on Si-terminated $\mathrm{SiC}(0001)$ surface. $J$ Appl Phys, 2014, 116(8):083502.

\section{SI TABLES}

Table S1.

Summary of the topological signatures and electronic properties of some currently identified two dimensional (2D) carbon allotropes, showing that hexagons are the primary building blocks of many of these materials, while carbon $2 \mathrm{D}$ structures made exclusively of pentagons are not known.

\begin{tabular}{|c|c|c|c|}
\hline 2D carbon structures & Carbon rings & Lattice $^{a}$ & Electronic property ${ }^{b}$ \\
\hline $\begin{array}{l}\text { graphene [1] } \\
\text { Spirographite [2] }\end{array}$ & $\begin{array}{l}6 \\
6\end{array}$ & $\begin{array}{l}\mathrm{H} \\
\mathrm{O}\end{array}$ & $\begin{array}{l}\mathrm{D} \\
\mathrm{M}\end{array}$ \\
\hline a-graphyne [3] & 18 & $\mathrm{H}$ & D \\
\hline $\begin{array}{l}\text { graphyne [4] } \\
6,6,12 \text {-graphyne [3] }\end{array}$ & $\begin{array}{l}6+12 \\
6+12\end{array}$ & $\begin{array}{l}\mathrm{H} \\
\mathrm{O}\end{array}$ & $\begin{array}{l}S \\
D\end{array}$ \\
\hline$\delta$-graphyne [5] & $6+15$ & $\mathrm{H}$ & D \\
\hline$\beta$-graphyne [3] & $12+18$ & $\mathrm{H}$ & D \\
\hline $\begin{array}{l}\text { Planar C4 [6, 7] or } \\
\text { T-graphene [8] }\end{array}$ & $4+8$ & $\mathrm{~T}$ & M \\
\hline Pentaheptite $[6,9,10]$ & $5+7$ & $\mathrm{M}$ or $\mathrm{O}^{c}$ & M \\
\hline $\begin{array}{l}\text { OPZ-L }[6,11] \\
\text { OPZ-Z }[6,11]\end{array}$ & $\begin{array}{l}5+8 \\
5+8\end{array}$ & $\begin{array}{l}M \\
\mathrm{O}\end{array}$ & $\begin{array}{l}M \\
G\end{array}$ \\
\hline Fused-pentagon network [12] & $5+12$ & $\mathrm{H}$ & M \\
\hline C31-sheet $[6,13]$ & $3+9$ & $\mathrm{H}$ & M \\
\hline C41-sheet [13] & $4+7$ & T & M \\
\hline C63-sheet [13] & $3+6+8$ & $\mathrm{H}$ & M \\
\hline $\begin{array}{l}\text { biphenylene }[6,14] \text { or } \\
\text { net-C [15] } \\
\text { H-net [16] } \\
\text { net-W }[15,17]\end{array}$ & $\begin{array}{l}4+6+8 \\
4+6+8 \\
4+6+8\end{array}$ & $\begin{array}{l}0 \\
T \\
0\end{array}$ & $\begin{array}{l}M \\
S \\
M\end{array}$ \\
\hline S-graphene [17] & $4+6+10$ & $\mathrm{O}$ & D \\
\hline $\begin{array}{l}\text { BPC }[13,18] \text { graphenylene } \\
{[19]}\end{array}$ & $4+6+12$ & $\mathrm{H}$ & $\mathrm{s}$ \\
\hline $\begin{array}{l}\text { pza-C10 [20] } \\
\text { Heackelite [21] } \\
\text { Dimerite [22] }\end{array}$ & $\begin{array}{l}5+6+7 \\
5+6+7 \\
5+6+7\end{array}$ & $\begin{array}{c}\mathrm{M} \\
\mathrm{H} \text { or M } \\
\mathrm{H}\end{array}$ & $\begin{array}{l}\mathrm{S} \\
\mathrm{M} \\
\mathrm{M}\end{array}$ \\
\hline $\begin{array}{l}\text { Octite [23] } \\
\text { HOPG [24] }\end{array}$ & $\begin{array}{l}5+6+8 \\
5+6+8\end{array}$ & $\begin{array}{l}\circ \\
\circ\end{array}$ & $\begin{array}{l}S \\
M\end{array}$ \\
\hline C65-sheet [13] & $5+6+9$ & $\mathrm{H}$ & M \\
\hline
\end{tabular}

aConsidering the finite-thickness of some 2D carbon allotropes, we use 3D lattices wherein the $c$ axis is perpendicular to the basal plane of the carbon sheets. The " $\mathrm{H}$ ", "T", "O", and "M" represent hexagonal, tetragonal, orthogonal, and monoclinic, respectively.

bThe "S" " $M$ ", "G" and "D" represent semiconductor, metal, gapless semimetal, and Dirac " level in the electronic band structure of the carbon sheet.

cSymmetry depends on the topological arrangements.

1. Novoselov KS, et al. Electric field effect in atomically thin carbon films. Science, 2004, 306(5696):666-669.
2. Bucknum MJ, Hoffmann R. A hypothetical dense 3,4-connected carbon net and related $\mathrm{B}_{2} \mathrm{C}$ and $\mathrm{CN}_{2}$ nets built from 1,4-cyclohexadienoid units. J Am Chem Soc, 1994, 116(25):11456-11464.

3. Malko D, Neiss C, Viñes F, Görling A. Competition for graphene: graphynes with direction-dependent Dirac cones. Phys Rev Lett, 2012, 108(8):086804.

4. Narita N, Nagai S, Suzuki S, Nakao K. Optimized geometries and electronic structures of graphyne and its family. Phys Rev B, 1998, 58(16):11009-11014.

5. Zhao M, Dong W, Wang A. Two-dimensional carbon topological insulators superior to graphene. Sci Rep, 2013, 3:3532.

6. Merz KM, Hoffmann R, Balaban AT. 3,4-connected carbon nets: through-space and through-bond interactions in the solid state. J Am Chem Soc, 1987, 109(22):6742-6751.

7. Enyashin AN, Ivanovskii AL. Graphene allotropes. Phys Status Solidi B, 2011, 248(8):1879-1883.

8. Liu Y, Wang G, Huang Q, Guo L, Chen X. Structural and electronic properties of T-Graphene: a two-dimensional carbon allotrope with tetrarings. Phys Rev Lett, 2012, 108(22):225505.

9. Crespi VH, Benedict LX, Cohen ML, Louie SG. Prediction of a pure-carbon planar covalent metal. Phys Rev B, 1996, 53(20):R13303-R13305.

10. Deza M, Fowler PW, Shtogrin M, Vietze K. Pentaheptite modifications of the graphite sheet. J Chem Inf Comp Sci, 2000, 40(6):1325-1332.

11. Su C, Jiang H, Feng J. Two-dimensional carbon allotrope with strong electronic anisotropy. Phys Rev B, 2013, 87(7):075453.

12. Mina M, Susumu O. Two-dimensional $s p^{2}$ carbon network of fused pentagons: all carbon ferromagnetic sheet. Appl Phys Express, 2013, 6(9):095101.

13. Lu H, Li S-D. Two-dimensional carbon allotropes from graphene to graphyne. J Mater Chem C, 2013,1(23):3677-3680.

14. Hudspeth MA, Whitman BW, Barone V, Peralta JE. Electronic properties of the Biphenylene sheet and its onedimensional derivatives. ACS Nano, 2010, 4(8):4565-4570.

15. Wang X-Q, Li H-D, Wang J-T. Prediction of a new twodimensional metallic carbon allotrope. Phys Chem Chem Phys, 2013, 15(6):2024-2030.

16. Hu M, Shu Yu, Cui L, Xu B, Yua D, He J. Theoretical twoatom thick semiconducting carbon sheet. Phys Chem Chem Phys, 2014, 16:18118-18123.

17. Xu LC, et al. Two dimensional Dirac carbon allotropes from graphene. Nanoscale, 2014, 6(2):1113-1118.

18. Brunetto $G$, et al. Nonzero gap two-dimensional carbon allotrope from porous graphene. J Phys Chem C, 2012, 116(23):12810-12813.

19. Song Q, et al. Graphenylene, a unique two-dimensional carbon network with nondelocalized cyclohexatriene units. J Mater Chem C, 2013, 1(1):38-41.

20. Luo $\mathrm{X}$, et al. Two-dimensional superlattice: modulation of band gaps in graphene-based monolayer carbon superlattices. J Phys Chem Lett, 2012, 3(22):3373-3378.

21. Terrones H, et al. New metallic allotropes of planar and tubular carbon. Phys Rev Lett, 2000, 84(8):1716-1719.

22. Lusk MT, Carr LD. Creation of graphene allotropes using patterned defects. Carbon, 2009, 47(9):2226-2232.

23. Appelhans DJ, Lin Z, Lusk MT. Two-dimensional carbon semiconductor: density functional theory calculations. Phys Rev B, 2010, 82(7):073410.

24. Mandal B, Sarkar S, Pramanik A, Sarkar P. Theoretical prediction of a new two-dimensional carbon allotrope and NDR behaviour of its one-dimensional derivatives. Phys Chem Chem Phys, 2013, 15(48):21001-21006. 
Table S2 Diameter (d) and band gap ( $\left.E_{q}\right)$ at the GGA/PBE level for the $(n, n)$ penta-tubes.

\begin{tabular}{|l|c|c|c|c|c|c|c|}
\hline \multicolumn{1}{|c|}{$\mathrm{n}$} & 2 & 3 & 4 & 5 & 6 & 7 & 8 \\
\hline $\mathrm{d}(\AA)$ & 3.559 & 5.089 & 6.734 & 8.354 & 9.979 & 11.609 & 13.308 \\
\hline $\mathrm{E}_{\mathrm{g}}(\mathrm{eV})$ & 1.783 & 2.339 & 2.540 & 2.608 & 2.514 & 2.474 & 2.334 \\
\hline
\end{tabular}

Table S3

Structural parameters of the tetragonal T12-carbon phase and its counterparts derived from the penta-graphene sheet.

\begin{tabular}{|l|l|l|l|}
\hline Structure & Space group & $\begin{array}{l}\text { Lattice } \\
\text { parameters }\end{array}$ & $\begin{array}{l}\text { Wyckoff } \\
\text { Atomic Positions }\end{array}$ \\
\hline T12 & P42/ncm (138) & $\begin{array}{l}\mathrm{a}=3.43 \AA \\
\mathrm{c}=6.09 \AA\end{array}$ & $\begin{array}{l}8 \mathrm{i}(0.3319,0.8319,0.3597) \\
4 \mathrm{~b}(0,0,0)\end{array}$ \\
\hline AA-T12 & P42/mnm (136) & $\mathrm{a}=3.52 \AA$ & $4 \mathrm{~d}(0,1 / 2,1 / 4)$ \\
& & $\mathrm{c}=6.29 \AA$ & $8 \mathrm{j}(0.8451,0.8451,0.6244)$ \\
\hline & & & $2 \mathrm{a}(0,0,0)$ \\
& & & $2 \mathrm{~b}(0,0,1 / 2)$ \\
ABAA-T24 & P-421m (113) & $\mathrm{a}=3.48 \AA$ & $4 \mathrm{e}(0.8316,0.3316,0.0686)$ \\
& & $\mathrm{c}=12.39 \AA$ & $4 \mathrm{e}(0.3435,0.8435,0.5645)$ \\
& & & $4 \mathrm{e}(0.3330,0.8330,0.1762)$ \\
& & & $4 \mathrm{e}(0.8426,0.6573,0.3093)$ \\
& & & $4 \mathrm{~d}(0.5000,0.5000,0.2454$ \\
\hline
\end{tabular}

ACKNOWLEDGMENTS. The authors thank the crew of the Center for Computational Materials Science, the Institute for Materials Research, Tohoku University (Japan), for their continuous support of the HITACHSR11000 supercomputing facility. This work is partially supported by grants from the National Natural Science Foundation of China (NSFC-51471004, NSFC-11174014, NSFC10990104, and NSFC-11334008), the National Grand Fundamental Research 973 Program of China (Grant 2012CB921404), and the Doctoral Program of Higher Education of China (20130001110033). S.Z. acknowledges funding from the Graduate School of Peking University that enabled him to visit P.J.'s group at Virginia Commonwealth University, where the present work is partially conducted. P.J. acknowledges support of the US Department of Energy, Office of Basic Energy Sciences, Division of Materials Sciences and Engineering under Award DE-FG02-96ER45579.

\section{REFERENCES}

1. KrotoHW,HeathJR, O'BrienSC, CurlRF,SmalleyRE. $\mathrm{C}_{60}$ : Buckminsterfullerene. Nature, 1985, 318(6042):162-163.

2. Iijima S, Ichihashi T. Single-shell carbon nanotubes of 1-nm diameter. Nature, 1993, 363(6430):603-605.

3. Novoselov KS, et al. Electric field effect in atomically thin carbon films. Science, 2004, 306(5696):666-669.

4. Charlier J-C, Rignanese G-M. Electronic structure of carbon nanocones. Phys Rev Lett, 2001, 86(26):5970-5973.
5. Jin C, Lan H, Peng L, Suenaga K, Iijima S. Deriving carbon atomic chains from graphene. Phys Rev Lett, 2009, 102(20):205501.

6. Li Y, Xu L, Liu H, Li Yu. Graphdiyne and graphyne: From theoretical predictions to practical construction. Chem Soc Rev, 2014, 43(8):2572-2586.

7. Bucknum MJ, Hoffmann R. A hypothetical dense 3,4-connected carbon net and related $\mathrm{B}_{2} \mathrm{C}$ and $\mathrm{CN}_{2}$ nets built from 1,4-cyclohexadienoid units. J Am Chem Soc, 1994, 116(25):11456-11464.

8. Zhang S, Wang Q, Chen X, Jena P. Stable threedimensional metallic carbon with interlocking hexagons. Proc Natl Acad Sci USA, 2013, 110(47):18809-18813.

9. Malko D, Neiss C, Viñes F, Görling A. Competition for graphene: Graphynes with direction-dependent Dirac cones. Phys Rev Lett, 2012, 108(8):086804.

10. Mina M, Susumu O. Two-dimensional $s p^{2}$ carbon network of fused pentagons: All carbon ferromagnetic sheet. Appl Phys Express, 2013, 6(9):095101.

11. Terrones $\mathrm{H}$, et al. New metallic allotropes of planar and tubular carbon. Phys Rev Lett, 2000, 84(8):1716-1719.

12. Xu L-C, et al. Two dimensional Dirac carbon allotropes from graphene. Nanoscale, 2014, 6(2):1113-1118.

13. Omachi H, Nakayama T, Takahashi E, Segawa Y, Itami K. Initiation of carbon nanotube growth by well-defined carbon nanorings. Nat Chem, 2013, 5(7):572-576.

14. Deza M, Fowler PW, Shtogrin M, Vietze K. Pentaheptite modifications of the graphite sheet. $J$ Chem Inf Comput Sci, 2000, 40(6):1325-1332.

15.15. Tan Y-Z, Xie S-Y, Huang R-B, Zheng L-S. The stabilization of fused-pentagon fullerene molecules. Nat Chem, 2009, 1(6):450-460.

16. Prinzbach H, et al. Gas-phase production and photoelectron spectroscopy of the smallest fullerene, $\mathrm{C}_{20}$. Nature, 2000, 407(6800):60-63.

17. Wang Y, et al. Template effect in the competition between Haeckelite and graphene growth on $\mathrm{Ni}(111)$ : Quantum chemical molecular dynamics simulations. $J$ Am Chem Soc, 2011, 133(46):18837-18842.

18. Zhao Z, et al. Tetragonal allotrope of group 14 elements. J Am Chem Soc, 2012, 134(30):12362-12365.

19. Naguib M, Gogotsi Y. Synthesis of two-dimensional materials by selective extraction. Acc Chem Res, 2015, 48(1):128-135.

20. Naguib M, et al. Two-dimensional nanocrystals produced by exfoliation of $\mathrm{Ti}_{3} \mathrm{AlC}_{2}$. Adv Mater, 2011, 23(37):4248-4253.

21. Ressouche E, Simonet V, Canals B, Gospodinov M, Skumryev V. Magnetic frustration in an ironbased Cairo pentagonal lattice. Phys Rev Lett, 2009, 103(26):267204. 
22. Menéndez-Proupin E, Montero-Alejo AL, García de la Vega JM. Ultrathin carbon nanotube with single, double, and triple bonds. Phys Rev Lett, 2012,109(10):105501.

23. Merz KM, Hoffmann R, Balaban AT. 3,4-connected carbon nets: Through-space and through-bond interactions in the solid state. J Am Chem Soc, 1987, 109(22):6742-6751.

24. Schmidt CL, Dinnebier R, Wedig U, Jansen M. Crystal structure and chemical bonding of the high-temperature phase of $\mathrm{AgN}_{3}$. Inorg Chem, 2007, 46(3):907-916.

25. Sheng X-L, Yan Q-B, Ye F, Zheng Q-R, Su G. T-carbon: A novel carbon allotrope. Phys Rev Lett, 2011, 106(15):155703.

26. Andrew RC, Mapasha RE, Ukpong AM, Chetty N. Mechanical properties of graphene and boronitrene. Phys Rev B, 2012, 85(12):125428.

27. Marianetti CA, Yevick HG. Failure mechanisms of graphene under tension. Phys Rev Lett, 2010, 105(24):245502.

28. Si C, Duan W, Liu Z, Liu F. Electronic strengthening of graphene by charge doping. Phys Rev Lett, 2012, 109(22):226802.

29. Ding Y, Wang Y. Density functional theory study of the silicene-like $\mathrm{SiX}$ and $\mathrm{XSi}_{3}(\mathrm{X}=\mathrm{B}, \mathrm{C}, \mathrm{N}, \mathrm{Al}, \mathrm{P})$ honeycomb lattices: The various buckled structures and versatile electronic properties. I Phys Chem C, 2013, 117(35):18266-18278.

30. Lee C, Wei X, Kysar JW, Hone J. Measurement of the elastic properties and intrinsic strength of monolayer graphene. Science, 2008, 321(5887):385-388.

31. Burns S. Negative Poisson's ratio materials. Science, 1987, 238(4826):551.

32. Jiang J-W, Park HS. Negative Poisson's ratio in singlelayer black phosphorus. Nat Commun, 2014, 5:4727.

33. Greaves GN, Greer AL, Lakes RS, Rouxel T. Poisson's ratio and modern materials. Nat Mater, 2011, 10(11):823-837.

34. Schreiner PR, et al. Overcoming lability of extremely long alkane carbon-carbon bonds through dispersion forces. Nature, 2011, 477(7364):308-311.

35. Lu S, Wang Y, Liu H, Miao MS, Ma Y. Self-assembled ultrathin nanotubes on diamond (100) surface. Nat Commun, 2014, 5:3666.

36. Heyd J, Scuseria GE, Ernzerhof M. Hybrid functionals based on a screened Coulomb potential. J Chem Phys, 2003, 118(18):8207-8215.

37. Heyd J, Scuseria GE, Ernzerhof M. Erratum: "Hybrid functionals based on a screened Coulomb potential." J Chem Phys, 2006, 124(21):219906.

38. Savini G, Ferrari AC, Giustino F. First-principles prediction of doped graphane as a high-temperature electron-phonon superconductor. Phys Rev Lett, 2010, 105(3):037002.

39. Charlier J-C, Blase X, Roche S. Electronic and transport properties of nanotubes. Rev Mod Phys, 2007, 79(2):677-732.

40. Ayala P, Arenal R, Loiseau A, Rubio A, Pichler T. The physical and chemical properties of heteronanotubes. Rev Mod Phys, 2010, 82(2):1843-1885.

41. Mao WL, et al. Bonding changes in compressed superhard graphite. Science, 2003, 302(5644):425-427.

42. Murnaghan FD. The compressibility of media under extreme pressures. Proc Natl Acad Sci USA, 1944, 30(9):244-247.

43. Nguyen MC, Zhao X, Wang C-Z, Ho K-M. sp ${ }^{3}-$ hybridized framework structure of group-14 elements discovered by genetic algorithm. Phys Rev B, 2014, 89(18):184112.

44. Davydov IV, Podlivaev AI, Openov LA. Anomalous thermal stability of metastable $\mathrm{C}_{20}$ fullerene. Phys Solid State, 2005, 47(4):778-784.

45. Niu H, et al. Families of superhard crystalline carbon allotropes constructed via cold compression of graphite and nanotubes. Phys Rev Lett, 2012, 108(13):135501.

46. Blase X, Gillet P, San Miguel A, Mélinon P. Exceptional ideal strength of carbon clathrates. Phys Rev Lett, 2004, 92(21):215505.

47. Riedl C, Coletti C, Iwasaki T, Zakharov AA, Starke U. Quasi-free-standing epitaxial graphene on SiC obtained by hydrogen intercalation. Phys Rev Lett, 2009, 103(24): 246804.

48. Sołtys J, Piechota J, Ptasinska M, Krukowski S. Hydrogen intercalation of single and multiple layer graphene synthesized on Si-terminated SiC(0001) surface. J Appl Phys, 2014, 116(8):083502.

49. Kresse G, Furthmüller J. Efficient iterative schemes for $\mathrm{ab}$ initio total-energy calculations using a planewave basis set. Phys Rev B Condens Matter, 1996, 54(16):11169-11186.

50. Blöchl PE. Projector augmented-wave method. Phys Rev B Condens Matter, 1994, 50(24):17953-17979.

51.51. Perdew JP, Burke K, Ernzerhof M. Generalized gradient approximation made simple. Phys Rev Lett, 1996, 77(18):3865-3868.

52. Monkhorst HJ, Pack JD. Special points for Brillouinzone integrations. Phys Rev B, 1976, 13(12):5188-5192.

53. Nosé S. A unified formulation of the constant temperature molecular dynamics methods. I Chem Phys, 1984, 81(1):511-519.

54. Togo A, Oba F, Tanaka I. First-principles calculations of the ferroelastic transition between rutile-type and $\mathrm{CaCl}_{2}$-type $\mathrm{SiO}_{2}$ at high pressures. Phys Rev B, 2008, 78(13): 134106. 